\begin{tabular}{|c|l|}
\hline Title & $\begin{array}{l}\text { Electrodeposition of Flattened Cu Nanoclusters on a p-GaA s(001) Electrode Monitored by in situ Optical Second } \\
\text { Harmonic Generation }\end{array}$ \\
\hline Author(s) & Y agi, Ichizo; Idojiri, Satoru; A watani, Tadashi; U osaki, Kohei \\
\hline Citation & $\begin{array}{l}\text { Journal of Physical Chemistry B, 109(11), 5021-5032 } \\
\text { https:/doi.org/10.1021/jp045458s }\end{array}$ \\
\hline Issue Date & 2005-03-24 \\
\hline Doc URL & http://hdl.handle.net/2115/50224 \\
\hline Type & article \\
\hline File Information & JPCB109-11_5021-5032.pdf \\
\hline
\end{tabular}

Instructions for use 


\title{
Electrodeposition of Flattened Cu Nanoclusters on a p-GaAs(001) Electrode Monitored by in situ Optical Second Harmonic Generation
}

\author{
Ichizo Yagi, ${ }^{*}, \dagger,+$ Satoru Idojiri, ${ }^{\dagger}$ Tadashi Awatani, ${ }^{\dagger}$ and Kohei Uosaki $*, \dagger$ \\ Physical Chemistry Laboratory, Division of Chemistry, Graduate School of Science, Hokkaido University, \\ Sapporo 060-0810, Japan, and CREST, Japan Science and Technology Agency (JST), \\ Kawaguchi 332-0012, Japan
}

Received: October 5, 2004; In Final Form: January 5, 2005

\begin{abstract}
In situ optical second harmonic generation (SHG) technique was employed to investigate the shape and density of $\mathrm{Cu}$ nanoclusters, which were electrochemically formed on $\mathrm{p}-\mathrm{GaAs}(001)$ electrode surfaces. Since GaAs is not a centrosymmetric medium, a significant portion of SHG signal arises from the bulk dipole susceptibility, but it was possible to separate a surface-induced signal from a bulk-induced signal by choosing an appropriate experimental geometry and appropriate data processing. The rotational anisotropy (RA) pattern of the SHG signal from a p-GaAs(001) electrode changed in both shape and magnitude during potential cycling in an electrolyte solution containing $\mathrm{Cu}^{2+}$. The surface plasmon-induced SHG signal from $\mathrm{Cu}$ nanoclusters deposited on GaAs was attributed to the modulation source for the RA-SHG pattern. More detailed study was carried out with both in situ SHG and ex situ AFM measurements for $\mathrm{Cu}$ nanoclusters deposited by potential step. The results showed that the SHG signal at the present optical geometry was sensitive to the number of oblate or flattened $\mathrm{Cu}$ nanoclusters with lateral diameter larger than $30 \mathrm{~nm}$ and that the SHG enhancement occurred because of resonant coupling between the surface plasmon induced in the flattened $\mathrm{Cu}$ nanoclusters and the near-infrared fundamental light.
\end{abstract}

\section{Introduction}

Metal particle growth on a semiconductor substrate has been an important issue for electronic device construction. Electrochemical deposition has been used to form a fine metallic structure. Gallium arsenide (GaAs) is one of the most important semiconductors in the high-frequency region because of its large carrier mobility. In our laboratory, electrodeposition of copper on a p-type $\mathrm{GaAs}(001)$ electrode has been investigated by using atomic force microscopy (AFM), ${ }^{1-4}$ extended X-ray absorption fine structures (EXAFS), ${ }^{5,6}$ and optical reflectance/scattering ${ }^{7}$ measurements. These studies covered the $\mathrm{Cu}$ deposition processes from atomic scale to micrometer scale, and information on the mechanism of $\mathrm{Cu}$ electrodeposition has been obtained. However, for the nanostructure formed before the completion of continuous $\mathrm{Cu}$ films, the relationship between deposition potential and characteristics of the $\mathrm{Cu}$ nanostructures, such as their size and shapes, has still been uncertain. By using linear optical spectroscopy, such as reflection and scattering measurements at a single optical wavelength,, 8 a rough picture of the surface structure can be obtained, but detailed characterization of $\mathrm{Cu}$ nanoclusters has not been realized. Although extinction and scattering spectroscopic measurements are the most useful approach for this purpose, difficulties in measurements and analyses for such spectroscopy often prevent us from obtaining a systematic view of the interfacial nanostructure formation. A simpler but powerful optical method is needed for the development of nanotechnology and surface science.

* Authors to whom correspondence should be addressed. E-mail: yagi@ pchem.sci.hokudai.ac.jp (I.Y.), uosaki@ PCL.sci.hokudai.ac.jp (K.U.). Fax: +81-11-706-3440. Phone: +81-11-706-3278.

$\dagger$ Hokkaido University.

$\doteqdot$ CREST
Nonlinear optical methods, ${ }^{9-14}$ such as optical second harmonic generation (SHG) and sum frequency generation (SFG), have been proved to be useful for application to chemistry and physics in nanoscale science and technology. SHG is one of the second-order nonlinear optical effects and is the conversion of two photons with frequency $\omega$ into one photon with a frequency of $2 \omega$. This phenomenon requires a lack of inversion symmetry, and in two neighboring centrosymmetric media, second harmonic $(\mathrm{SH})$ light can be generated only from a few atomic or molecular layers at the interface. Thus, the SHG signal is usually recognized to be inherently surface sensitive, and various kinds of information at the interface can be extracted by changing the measurement parameters. ${ }^{15-18}$

Compound semiconductors are noncentrosymmetric and bulk contribution to SHG is present. It is well-known that GaAs is one of the materials having large second-order susceptibilities. ${ }^{19}$ To monitor the surface reaction at GaAs surfaces by SHG, it is necessary to establish a method by which the surface and bulk contributions can be separated. Sterhlin et al. observed the surface contribution to SHG signals from GaAs(001) and showed that the bulk contribution can be canceled by using the appropriate combination of light polarizations and the azimuthal angle of $\mathrm{GaAs}(001) \cdot{ }^{20}$ Yamada and Kimura developed a method to analyze the SHG rotational anisotropic patterns to separate the bulk and surface contributions, and they also confirmed that the surface contribution to the SHG signal apparently became larger because of the interference between the surface and bulk contributions in the case of the $\mathrm{p}$-in/p-out polarization combination. ${ }^{21-25}$ By using this effect, Tanaka et al. obtained SHG spectra of a GaAs(001) surface showing a novel resonant feature. ${ }^{26}$ These studies on GaAs surfaces proved that the SHG method is a powerful tool to monitor surface or interfacial 
phenomena even with the existence of large bulk contributions to the signal. ${ }^{21-25,27-30}$

In the present study, both in situ SHG and ex situ AFM methods were used to characterize $\mathrm{Cu}$ nanoclusters electrochemically deposited on $\mathrm{n}-\mathrm{GaAs}(001)$ electrodes in the initial stages of $\mathrm{Cu}$ film formation on this substrate. The change in the SHG rotational anisotropy pattern during potential-cycling $\mathrm{Cu}$ deposition was followed, and then the surface SHG signal, which seemed to be sensitive to the $\mathrm{Cu}$ nanocluster itself, was monitored during the potentiostatic $\mathrm{Cu}$ deposition. Only the $\mathrm{Cu}$ nanoclusters deposited at a specific potential region showed enhancement of the surface SHG signal. By characterization of the resulting surfaces with ex situ AFM, the surface SHG enhancement was attributed to flattened $\mathrm{Cu}$ nanoclusters with specific sizes on $\operatorname{GaAs}(001)$.

\section{Experimental Section}

Ultrapure-grade $\mathrm{H}_{2} \mathrm{SO}_{4}$, ethanol, and reagent-grade $\mathrm{CuSO}_{4}$ were obtained from Wako Pure Chemicals and used without further purification. Water was purified using a Milli-Q water purification system (Yamato, WQ-500).

Single crystalline Zn-doped p-type $\operatorname{GaAs}(001)$ wafers (Mitsubishi Chemical Corp., doping density of $8-30 \times 10^{18} \mathrm{~cm}^{-3}$ ) were used as sample electrodes. The samples were cleaned in hot acetone and hot ethanol and then chemically etched in $3 \mathrm{M}$ $\mathrm{HCl}$ aqueous solution for $30 \mathrm{~s}$ and rinsed with Milli-Q water. Ohmic contact was secured using an InZn alloy. The sample was fixed on a spectroelectrochemical cell, which was made from Kel-F with an optically flat fused silica window. The electrode potential was controlled by an electric polarization unit (Toho Technical Research, PS-07) with respect to the Ag/ $\mathrm{AgCl}$ electrode (saturated $\mathrm{NaCl}$ ), and the counter electrode was a Pt wire. After sample preparation and setting of a spectroelectrochemical cell, the deaerated electrolyte solution was introduced into the cell with $\mathrm{Ar}$ gas pressure at the sample potential of $+100 \mathrm{mV}$, where neither anodic oxidation of the GaAs surface nor electrodeposition of $\mathrm{Cu}$ occurred. The electrolyte solution was $0.1 \mathrm{M}$ of $\mathrm{H}_{2} \mathrm{SO}_{4}$, and for the electrochemical deposition of $\mathrm{Cu}, \mathrm{CuSO}_{4}$ was dissolved in this solution to the concentration of $1 \mathrm{mM}$.

SHG measurement was carried out by using a fundamental light $(\lambda=1064 \mathrm{~nm})$ from an Nd:YAG laser (Coherent, Infinity 40-100). The repetition rate and the pulse width of the laser pulses were $20 \mathrm{~Hz}$ and about $2 \mathrm{~ns}$, respectively. The fundamental beam was slightly focused on the sample surface in the spectroelectrochemical cell, which was mounted on an $x, y, z$, and $\theta$ translational stage, and the generated SHG beam of 532 $\mathrm{nm}$ was separated from the reflection through filters (Toshiba Filter, IRA-20) and a monochromator (Koken, SG-100), and the beam was then detected by a photomultiplier tube (Hamamatsu Photonics, R636-10). The incident angle of the fundamental beam was $\sim 42^{\circ}$, and the input and output polarizations were selected by using appropriate polarizers. The output of the photomultiplier tube was averaged over 30 laser shots with gated electronics and then was captured by a computer through a 12 bit A/D converter. SH rotational anisotropy ( $\mathrm{SH}-\mathrm{RA}$ ) measurement was carried out by recording the SH intensity during the sample rotation around the surface normal at fixed potential, and the azimuthal angle was defined as the angle between the plane of incidence and the [100] axis on the GaAs(001) surface as shown in Figure 1. Potential-dependent SHG measurement was carried out by recording $\mathrm{SH}$ intensity during potential cycling with a sweep rate of $5 \mathrm{mV} \mathrm{s}^{-1}$. For potential step measurement, the potential was controlled by a 12 bit D/A

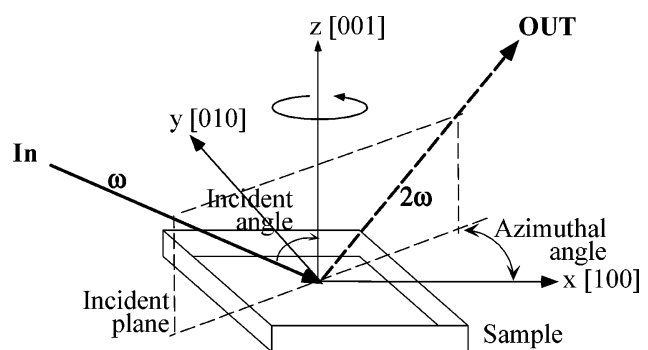

Figure 1. Optical arrangement for SHG rotational anisotropy (SHRA) measurements.

converter and potentiostat, and the potential, current, and SHG intensity during $\mathrm{Cu}$ deposition were recorded simultaneously.

Ex situ AFM measurements were carried out using a Nanoscope E (Digital Instruments) in a constant-force mode. The spring constant of the cantilevers was $0.12 \mathrm{~N} \mathrm{~m}^{-1}$. The samples for ex situ AFM measurements were prepared by potential-step deposition with the same electrochemical cell, and Faradaic charge density was integrated during $\mathrm{Cu}$ deposition. When Faradaic charge density reached a desirable value, contact between the GaAs electrode and electrolyte solution was removed, and then the surface was rinsed with water and dried with an Ar gas flow. The samples were immediately transferred to an AFM stage.

\section{Results}

3.1. SH Rotational Anisotropy Patterns of a Bare p-GaAs(001) Surface. Figure 2 shows SH-RA patterns of a chemically etched bare $\mathrm{p}-\mathrm{GaAs}(001)$ surface measured in air with various polarization combinations. For (b) p-in/s-out and (c) s-in/p-out polarization combinations, four-fold symmetry patterns reflecting $C_{4 v}$ symmetry of GaAs bulk were observed. These four peaks originate from the nonlinearity of $-\mathrm{Ga}-\mathrm{As}-\mathrm{Ga}-\mathrm{As}-$ chains in the bulk structure in [110] and [1 10$]$ directions. On the other hand, a two-fold symmetry pattern was obtained in the case of a $\mathrm{p}$-in/p-out polarization combination. These $\mathrm{SH}$ RA patterns are fitted by the following equation: ${ }^{22,26}$

$$
I(2 \omega)=|A+B \sin (2 \phi)|^{2}
$$

where $\phi$ is the azimuthal angle, which is defined as the angle between the optical incident plane and [100] axis on the GaAs(001) surface, and $A$ and $B$ are the isotropic and anisotropic responses of $\mathrm{SHG}$, respectively.

As has been reported previously, ${ }^{22,26}$ the two-fold symmetry observed only in the case of a $\mathrm{p}$-in/p-out polarization combination was due to the interference between the isotropic surface contribution and $C_{4 v}$ bulk contribution. This isotropic surface contribution arises from the nonlinear polarization induced by the optical process, represented by the surface nonlinear susceptibility elements, $\chi_{\mathrm{s}}^{(2)} z z z$ or $\chi_{\mathrm{s}}^{(2)} x z x=\chi_{\mathrm{s}}^{(2)} y z y$. Thus, the change in the isotropic surface contribution results in modification of the shape in the p-in/p-out SH-RA pattern, and only SHG measurement in the $\mathrm{p}$-in/p-out polarization combination can detect the surface event directly. By utilizing the interference between the surface and bulk contributions, the surface reconstruction $^{22}$ and surface-localized resonance ${ }^{26}$ were detected at GaAs(001).

3.2. Potential-Dependent SHG Profiles at a Bare p-GaAs(001) Electrode. First, the potential dependencies of current and $\mathrm{p}$-in/p-out $\mathrm{SH}$ intensity at a bare $\mathrm{p}-\mathrm{GaAs}(001)$ electrode in a $0.1 \mathrm{M} \mathrm{H}_{2} \mathrm{SO}_{4}$ solution were measured. In a cyclic voltammogram (CV) (Figure 3a), almost no Faradaic current was observed in the potential region between -500 and $+100 \mathrm{mV}$. 


\section{(a)p-in/p-out}
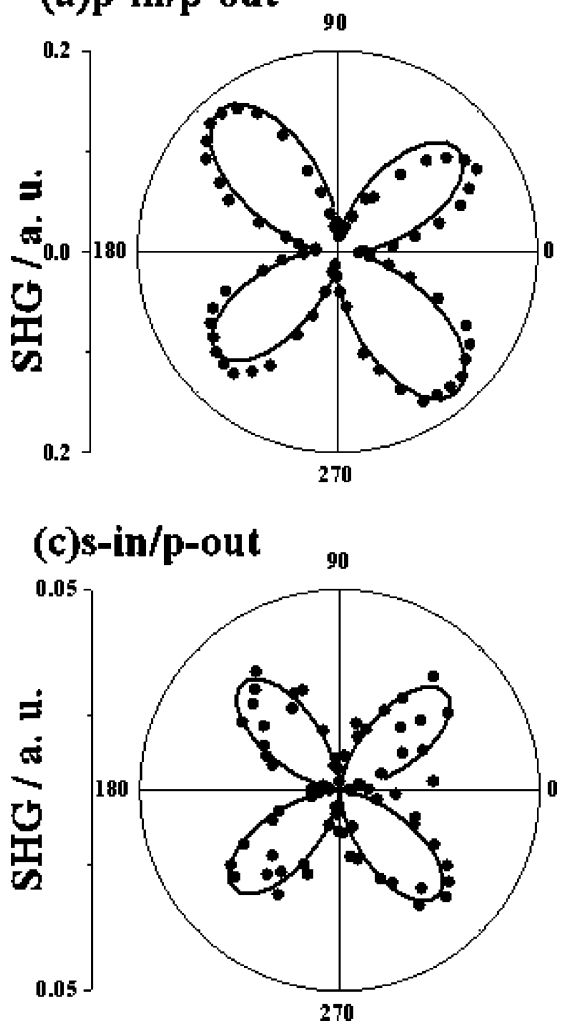

\section{(b)p-in/s-out}

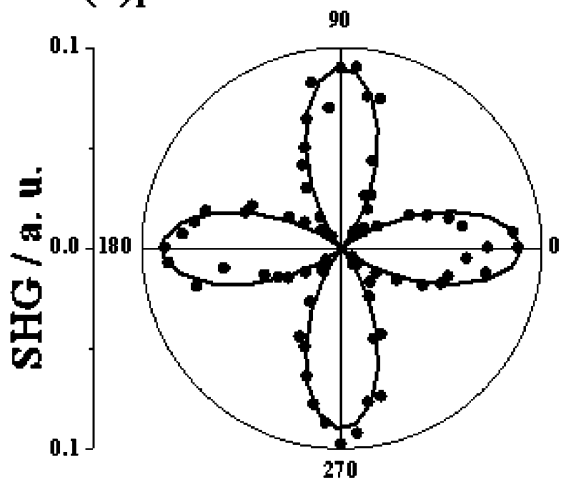

[010]

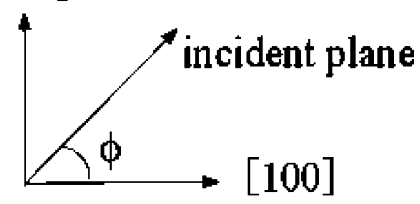

Figure 2. SHG rotational anisotropy (SH-RA) patterns of p-GaAs(001) surface in air for various polarization combinations: (a) p-in/p-out, (b) p-in/s-out, and (c) s-in/p-out.

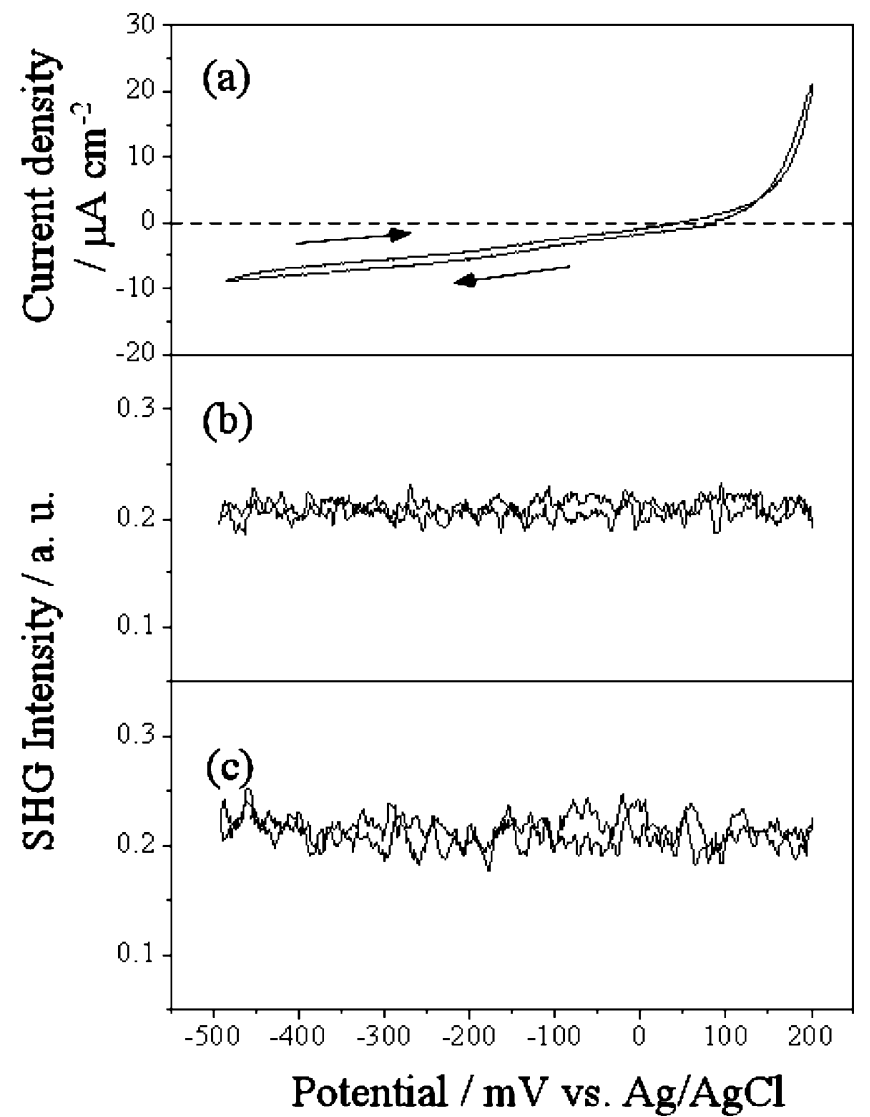

Figure 3. Potential dependences of (a) current and p-in/p-out SHG intensities at (b) $\phi=45^{\circ}$ and (c) $\phi=135^{\circ}$ in $0.1 \mathrm{M} \mathrm{H}_{2} \mathrm{SO}_{4}$ solution. Scan rate $=5 \mathrm{mV} \mathrm{s}^{-1}$.

Since the p-in/p-out $\mathrm{SH}$ intensities at the azimuthal angles of $45^{\circ}$ (Figure $3 \mathrm{~b}$ ) and $135^{\circ}$ (Figure $3 \mathrm{c}$ ), where lower and higher

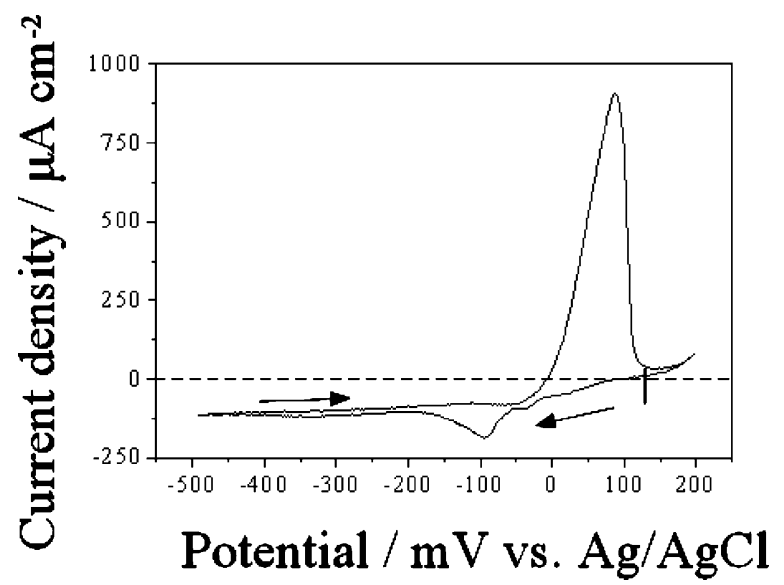

Figure 4. Cyclic voltammogram of p-GaAs(001) electrode in $0.1 \mathrm{M}$ $\mathrm{H}_{2} \mathrm{SO}_{4}$ solution containing $1 \mathrm{mM}$ of $\mathrm{CuSO}_{4}$. Sweep rate $=5 \mathrm{mV} \mathrm{s}^{-1}$.

peaks were observed in the $\mathrm{p}$-in/p-out SH-RA pattern, showed no change during a potential sweep, it is clear that the potential has no effect on $\mathrm{SH}$ intensities in a $0.1 \mathrm{M} \mathrm{H}_{2} \mathrm{SO}_{4}$ solution. Thus, the electric field-induced SHG (EFISH) can be neglected in the present measurement conditions, reflecting the dominant contribution from GaAs bulk. The EFISH effect has been reported to be wavelength dependent and to be small at an excitation of $1064 \mathrm{~nm}$ at GaAs(111)B surfaces. ${ }^{31}$ In other polarization combinations, constant SH intensity was also observed in the same potential region.

3.3. Potential Sweep Measurements in a Cu-Containing Solution. Figure 4 shows a CV of a p-GaAs(001) electrode in a $0.1 \mathrm{M} \mathrm{H}_{2} \mathrm{SO}_{4}$ solution containing $1 \mathrm{mM}$ of $\mathrm{CuSO}_{4}$. In the negative potential scan from $+100 \mathrm{mV}$, a cathodic current started to flow from $\sim-60 \mathrm{mV}$, reached a cathodic peak at around $-100 \mathrm{mV}$, and decreased to a relatively constant value 
(a) $+100 \mathrm{mV}$

(before potential sweep)

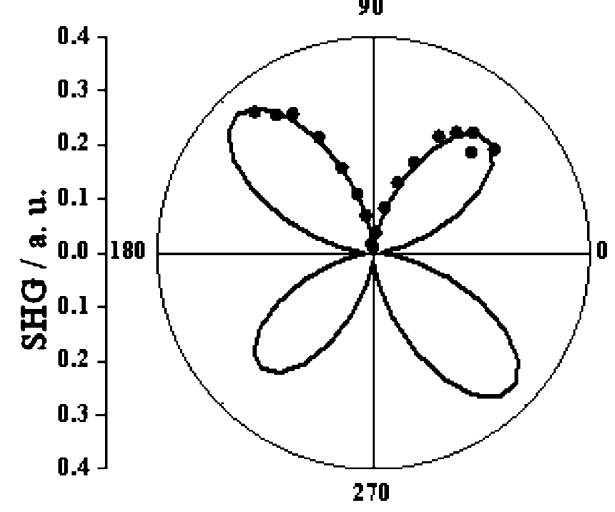

(c) $0 \mathrm{mV}$

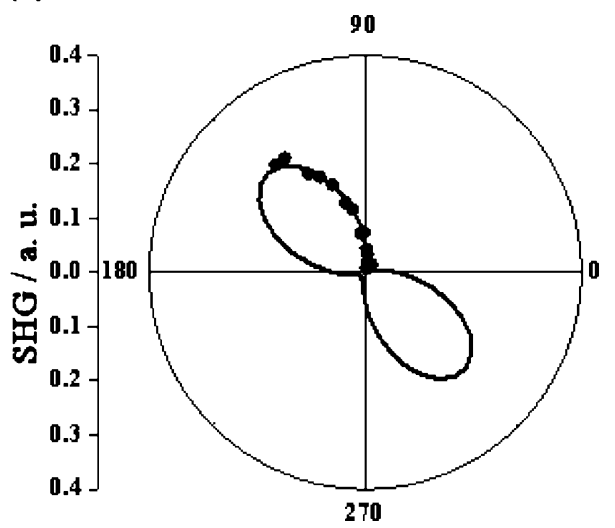

(b) $-500 \mathrm{mV}$

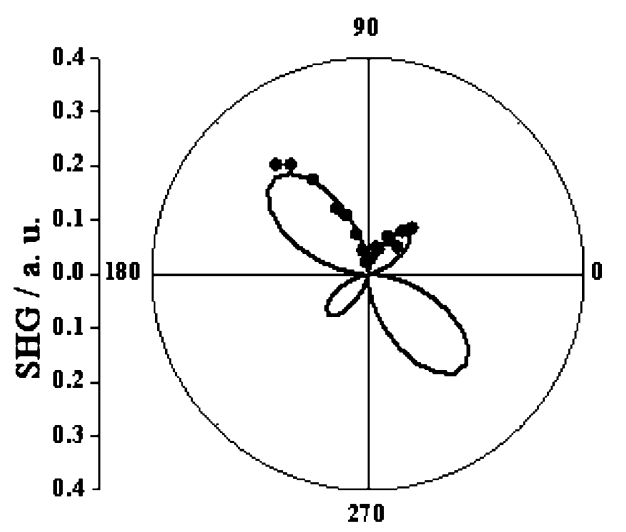

(d) $+100 \mathrm{mV}$ (anode peak)

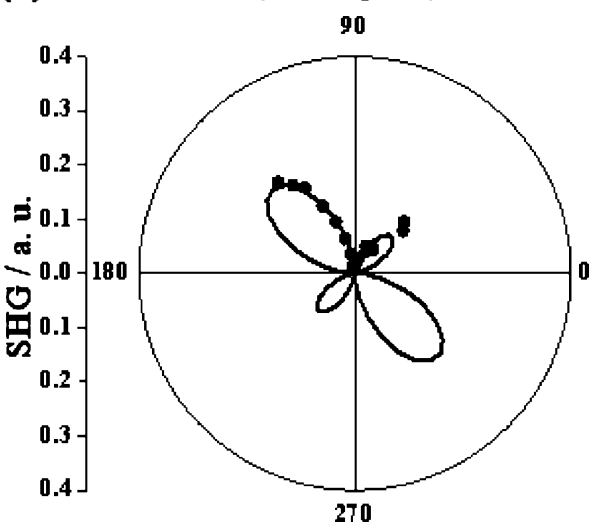

Figure 5. SH-RA patterns of $\mathrm{p}-\mathrm{GaAs}(001)$ electrode in $0.1 \mathrm{M} \mathrm{H}_{2} \mathrm{SO}_{4}$ solution containing $1 \mathrm{mM}$ of $\mathrm{CuSO}_{4}$ at various fixed potentials: (a) +100 (before potential sweep), (b) -500 , (c) 0 (in the positive potential sweep), and (d) $+100 \mathrm{mV}$ (at anode peak).

corresponding to the diffusion-limited current. The cathodic current was due to the deposition of $\mathrm{Cu}$ on the p-GaAs(001) electrode, and a diffusion-limited deposition current was still observed even in the positive-going potential scan from -500 $\mathrm{mV}$. The anodic current corresponding to the dissolution of the deposited $\mathrm{Cu}$ started to flow from around $0 \mathrm{~V}$ with an anodic peak at $+100 \mathrm{mV}$. The values of the integrated Faradaic charge for deposition and dissolution currents were almost the same, and the same $\mathrm{CV}$ was obtained in the following potential cycles, showing reversible deposition/dissolution reactions of $\mathrm{Cu}$ on p-GaAs(001). The shape of CV was almost the same as those obtained in previous studies. ${ }^{2,5,7}$

As shown in Figure 5, the shape and amplitude of the p-in/ p-out SH-RA pattern changed corresponding to the deposition/ dissolution processes. At the initial potential of $+100 \mathrm{mV}$, almost the same SH-RA pattern as that in air (Figure 2a) was obtained. The filled circles in the p-in/p-out SH-RA patterns during potential cycling in Figure 5 were constructed from the potential-dependent SHG profiles at fixed azimuthal angles from $60^{\circ}$ to $120^{\circ}$, and the solid lines are the result of fitting for the SH-RA patterns by eq 1 since in situ SH-RA measurements at fixed negative potentials in $\mathrm{Cu}$-containing solution were impossible because of the continuous $\mathrm{Cu}$ deposition. After several potential cycles, almost the same SH-RA pattern was obtained at $+100 \mathrm{mV}$, confirming the reversible deposition/dissolution cycles of $\mathrm{Cu}$ on $\mathrm{p}-\mathrm{GaAs}(001)$. In the $\mathrm{p}$-in/p-out SH-RA patterns shown in Figure 5, the lower peaks seemed to decrease faster than the higher peaks, and then the two-fold symmetric shape of the pattern became clearer with increase in the surface coverage of $\mathrm{Cu}$. It has been reported that these lower and higher peaks in the $\mathrm{p}$-in/p-out SH-RA pattern appeared as a result of destructive and constructive interference between the surface and bulk contributions, respectively. ${ }^{22,26,29}$ Thus, the modification of the shape in the p-in/p-out SH-RA pattern should be due to either decrease in bulk SHG or increase in surface SHG.

Figure 6 shows potential-dependent p-in/p-out SHG behaviors at fixed azimuthal angles of (b) $45^{\circ}$, (c) $90^{\circ}$, and (d) $135^{\circ}$ with spontaneously obtained (a) CV. These angles correspond to the azimuthal angle of the lower peak, zero contribution from bulk, and higher peak, respectively. Potential dependencies of p-in/ s-out SHG at the fixed azimuthal angles of (b) $0^{\circ}$, (c) $45^{\circ}$, and (d) $90^{\circ}$, which contain only the bulk contribution, were also obtained as shown in Figure 7 with (a) CV. In the p-in/s-out polarization combination, the $\mathrm{SH}$ intensities at the peak azimuthal angles (Figure $7 \mathrm{~b}$ and $\mathrm{d}$ ) showed only a monotonic decrease with increase in the surface coverage of $\mathrm{Cu}$. The $\mathrm{SH}$ intensity at the azimuthal angle of $45^{\circ}$ (Figure 7c), where no contribution from bulk was expected, showed no change during potential cycling. These behaviors indicate that the $\mathrm{p}$-in/s-out SH-RA pattern kept its shape with only a decrease in the amplitude during $\mathrm{Cu}$ deposition. On the other hand, the p-in/ p-out SH intensities at the peak azimuthal angles of $45^{\circ}$ (Figure $6 \mathrm{~b}$ ) and $135^{\circ}$ (Figure 6d) showed different potential-dependent behaviors, although these also decreased with increase in the $\mathrm{Cu}$ coverage. At the azimuthal angle of $90^{\circ}$ (Figure 6c), where the bulk contribution is zero and only the surface contribution should be monitored, the SH intensity showed a slight increase with increase in $\mathrm{Cu}$ coverage and returned to zero with the dissolution of $\mathrm{Cu}$. By comparing these figures, it is clear that the $\mathrm{p}$-in/p-out $\mathrm{SH}$ responses at the azimuthal angles of $45^{\circ}$ 


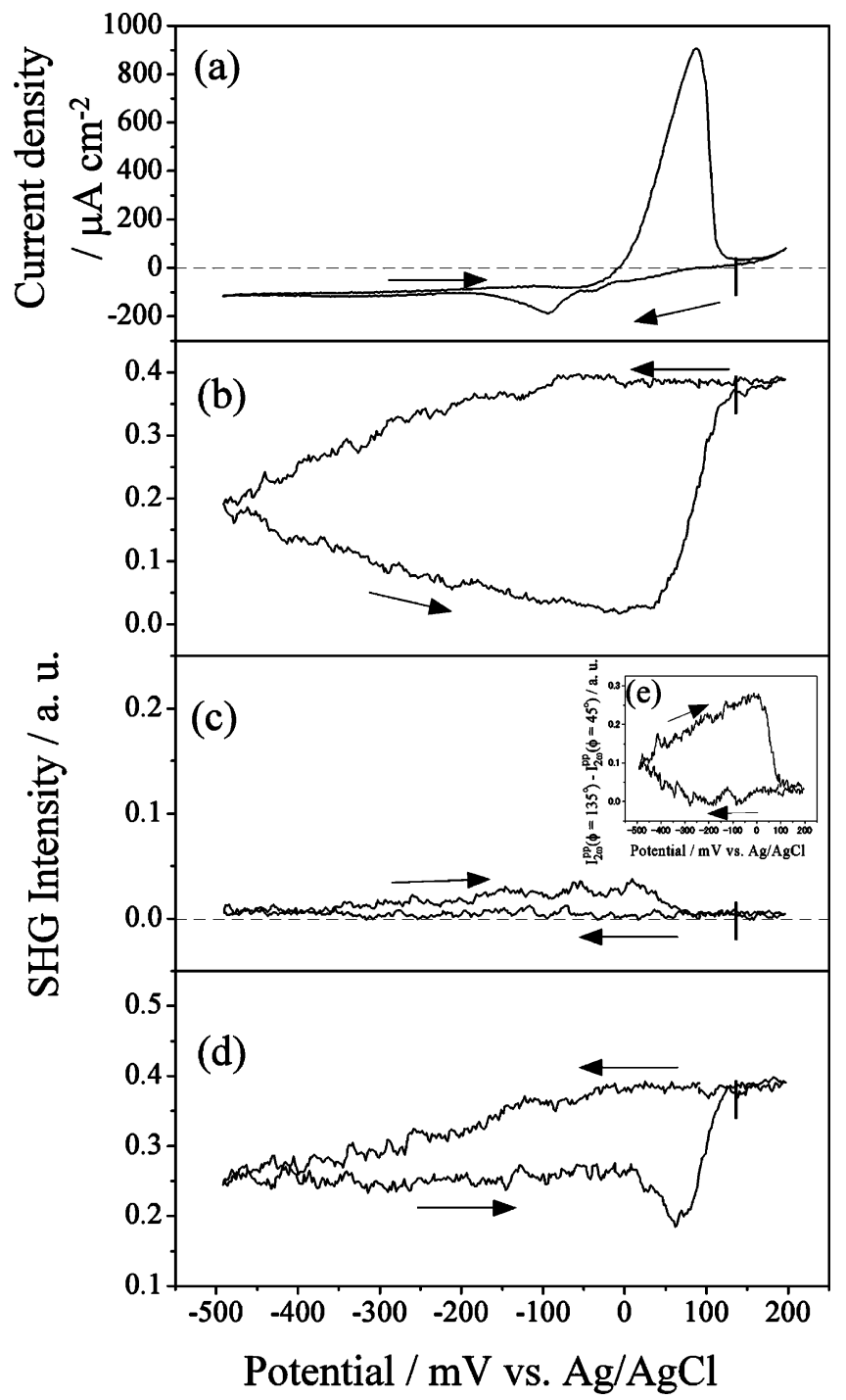

Figure 6. Potential dependences of (a) current and p-in/p-out SHG intensity at p- $\mathrm{GaAs}(001)$ electrode in $0.1 \mathrm{M} \mathrm{H}_{2} \mathrm{SO}_{4}$ solution containing $1 \mathrm{mM}$ of $\mathrm{CuSO}_{4}$; azimuthal angle, $\phi=$ (b) $45^{\circ}$, (c) $90^{\circ}$, and (d) $135^{\circ}$. (e) Potential dependence of subtracted surface SHG responses calculated from curves (b) and (d).

(Figure 6b) and $135^{\circ}$ (Figure 6d) can be reproduced by expansively adding the surface SHG (the p-in/p-out SHG at the azimuthal angle of $90^{\circ}$, Figure 6c) on the bulk SHG (the p-in/ s-out SHG at the azimuthal angle of $0^{\circ}$ or $90^{\circ}$, Figure $7 \mathrm{~b}$ or d) destructively and constructively, as has been described. ${ }^{22,26,29}$ Interference between surface SHG and bulk SHG can be shown more clearly by subtracting the $\mathrm{SH}$ intensity of the lower peak (at $\phi=45^{\circ}$ ) from that of the higher peak (at $\phi=135^{\circ}$ ) in the p-in/p-out SH-RA pattern. Figure $6 \mathrm{e}$ shows the potential dependence of the difference between the higher- and lowerpeak $\mathrm{SH}$ intensities calculated from Figure $6 \mathrm{~b}$ and $d$. The similarity between the potential dependence in Figure 6e and that in Figure $6 \mathrm{c}$ is obvious, and that of the former seemed more sensitive to the surface event than that of the latter. Yamada and Kimura suggested that the surface SHG signal was magnified by interference with the dipole-allowed bulk SHG in the p-in/p-out SH-RA pattern. ${ }^{22}$ The present results support their speculation. From the above results, the deposition of $\mathrm{Cu}$ caused both a decrease in bulk SHG and an increase in surface SHG, and then the p-in/p-out SH-RA pattern changed its shape with an increase in the surface coverage of $\mathrm{Cu}$.

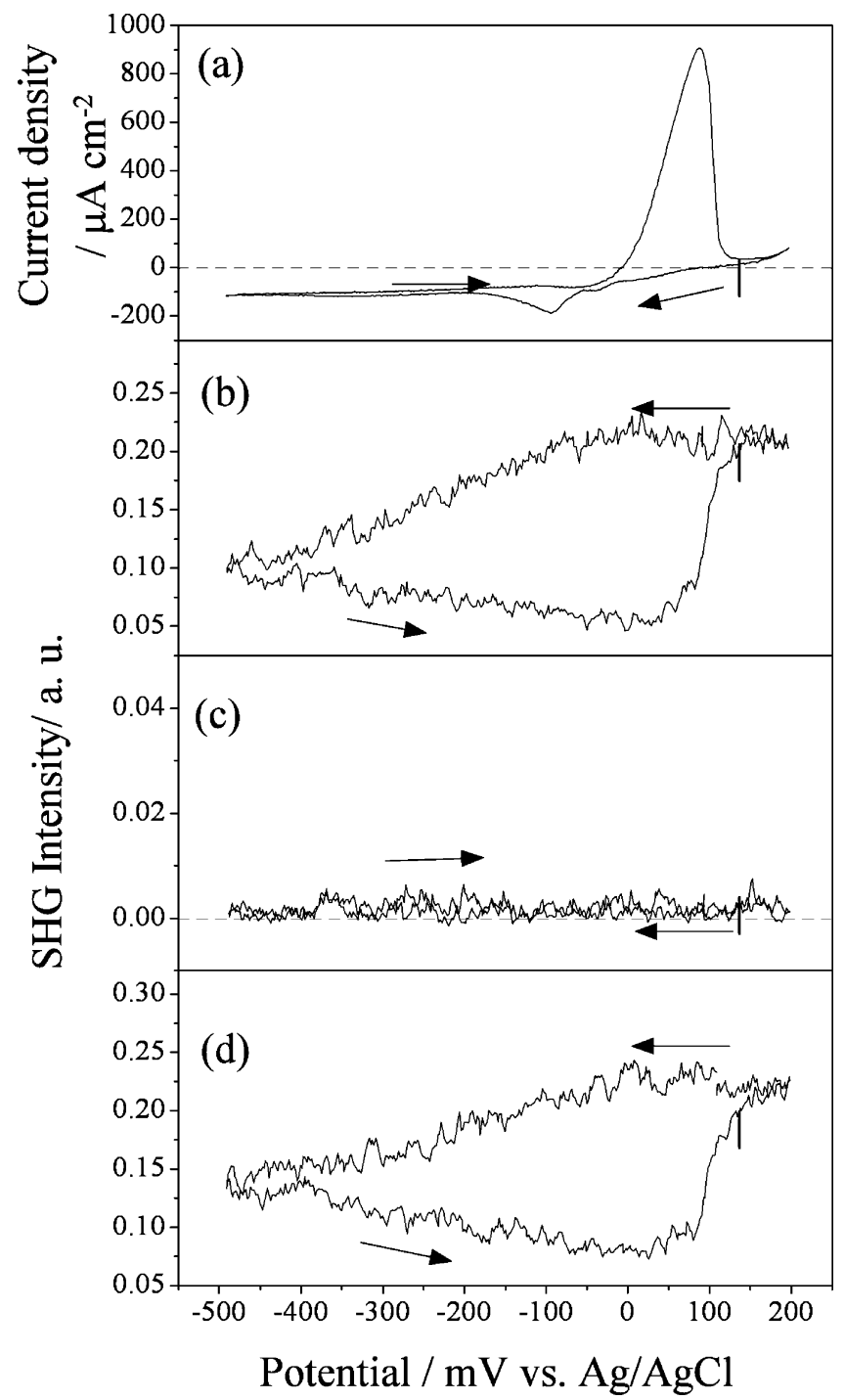

Figure 7. Potential dependences of (a) current and p-in/s-out SHG intensity at $\mathrm{p}-\mathrm{GaAs}(001)$ electrode in $0.1 \mathrm{M} \mathrm{H}_{2} \mathrm{SO}_{4}$ solution containing $1 \mathrm{mM}$ of $\mathrm{CuSO}_{4}$; azimuthal angle, $\phi=$ (b) $0^{\circ}$, (c) $45^{\circ}$, and (d) $90^{\circ}$.

3.4. Potential Step Experiments. In the above-described experiments, the potential was swept at the sweep rate of $5 \mathrm{mV}$ $\mathrm{s}^{-1}$. Since the number of nucleation sites for electrodeposition of $\mathrm{Cu}$ on a $\mathrm{p}-\mathrm{GaAs}(001)$ electrode has been shown to depend on overpotential, ${ }^{1,2,7} \mathrm{Cu}$ deposition by potential sweep caused progressive nucleation and growth, resulting in complicated morphology of $\mathrm{Cu}$ deposits with various cluster sizes. On the other hand, a number of $\mathrm{Cu}$ deposition/dissolution cycles or deposition of a large amount of $\mathrm{Cu}$ caused the formation of irreversibly deposited $\mathrm{Cu}$ sites, and the shape of $\mathrm{CV}$ gradually deformed during deposition/dissolution cycles. Although such deformation of $\mathrm{CV}$ (depending on the history of the sample) was avoided by carefully choosing a small deposition amount of $\mathrm{Cu}$ in the potential cycling measurements, completely clean $\mathrm{GaAs}$ samples could not be reproduced after $\mathrm{Cu}$ deposition cycles. To avoid the effect of the surface history for each deposition and to understand the mechanism of the increase in surface SHG by $\mathrm{Cu}$ deposition as shown in the previous section, in situ p-in/p-out SHG measurements at the azimuthal angle of $90^{\circ}$ on freshly prepared GaAs samples with potential step from the initial potential of $+100 \mathrm{mV}$ to several deposition potentials were carried out since potentiostatic deposition of $\mathrm{Cu}$ results in the instantaneous nucleation and growth of $\mathrm{Cu}$ deposits. 


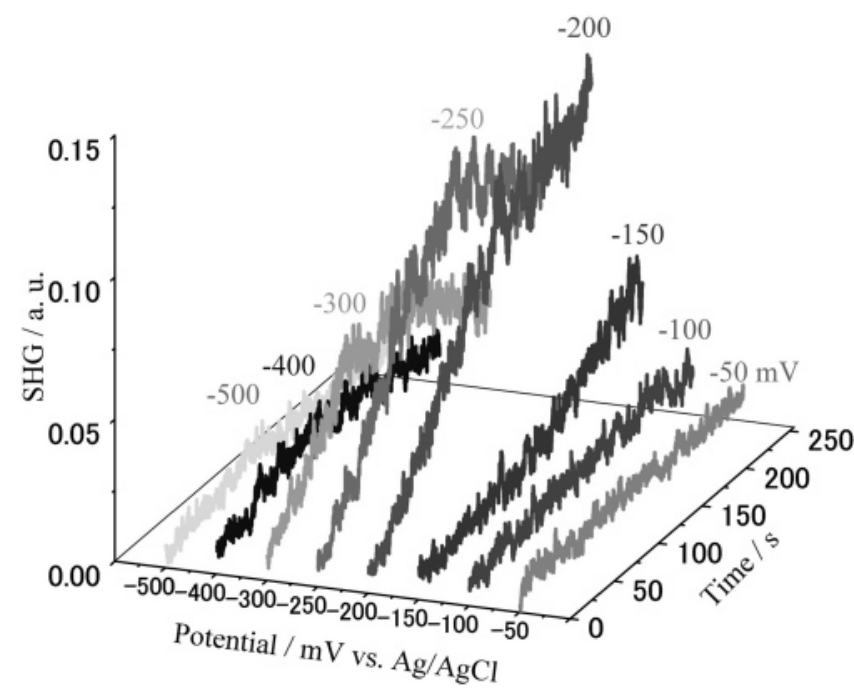

Figure 8. Three-dimensional plot of time-dependent p-in/p-out SHG intensity transients at p-GaAs(001) electrode in $0.1 \mathrm{M} \mathrm{H}_{2} \mathrm{SO}_{4}$ solution containing $1 \mathrm{mM}$ of $\mathrm{CuSO}_{4}$. Azimuthal angle was fixed at $90^{\circ}$. The electrode potential was stepped from $+100 \mathrm{mV}$ to each deposition potential at $t=0 \mathrm{~s}$.

Figure 8 shows the time-dependent p-in/p-out SH intensities at the azimuthal angle of $90^{\circ}$ during $\mathrm{Cu}$ deposition at various deposition potentials. In such an experimental arrangement, only the surface SHG can be monitored, as indicated above. As shown in Figure 8, an increase in surface SHG by $\mathrm{Cu}$ deposition was observed only at a few deposition potentials. The increase in surface SHG was largest at the deposition potential of -200 $\mathrm{mV}$, where the $\mathrm{SH}$ intensity kept increasing even after deposition for $250 \mathrm{~s}$. At the deposition potentials of -250 and $-300 \mathrm{mV}$, surface SHG response reached a maximum at a certain deposition time and then decreased. At the deposition potentials of -175 and $-150 \mathrm{mV}$, the increase in surface SHG response was slower than that at $-200 \mathrm{mV}$. At more negative and positive deposition potentials, no change was observed in surface SHG response.

Typical responses of surface SHG at seven deposition potentials are plotted against Faradaic charge in Figure 9a and b. Surface SHG response at the deposition potential of -200 $\mathrm{mV}$ is plotted both in Figure 9a and b for comparison, since the surface SHG behaviors during $\mathrm{Cu}$ deposition changed clearly around the deposition potential of $-200 \mathrm{mV}$. At potentials more negative than $-200 \mathrm{mV}$, surface SHG increased with the same slope against the Faradaic charge and started to decrease from a certain charge, depending on each deposition potential. On the other hand, at potentials more positive than $-200 \mathrm{mV}$, surface SHG increased with almost the same slope from a certain charge.

The bulk SHG behaviors during potentiostatic $\mathrm{Cu}$ deposition were also monitored by measuring the p-in/s-out SH intensity at the azimuthal angle of $0^{\circ}$. In Figure 9c, bulk SHG responses at several deposition potentials are plotted against the Faradaic charge. Although the surface SHG behavior depended on the deposition potential, all of the bulk SHG responses showed only a steady decrease with similar slopes as the $\mathrm{Cu}$ deposition charge increased. Even at the deposition potentials of -500 and -50 $\mathrm{mV}$, where no change in surface SHG was observed, bulk SHG decreased with the Faradaic charge. Thus, it can be concluded that the attenuation of bulk SHG from p-GaAs depended on the amount of $\mathrm{Cu}$ deposits and was independent of deposition potential.

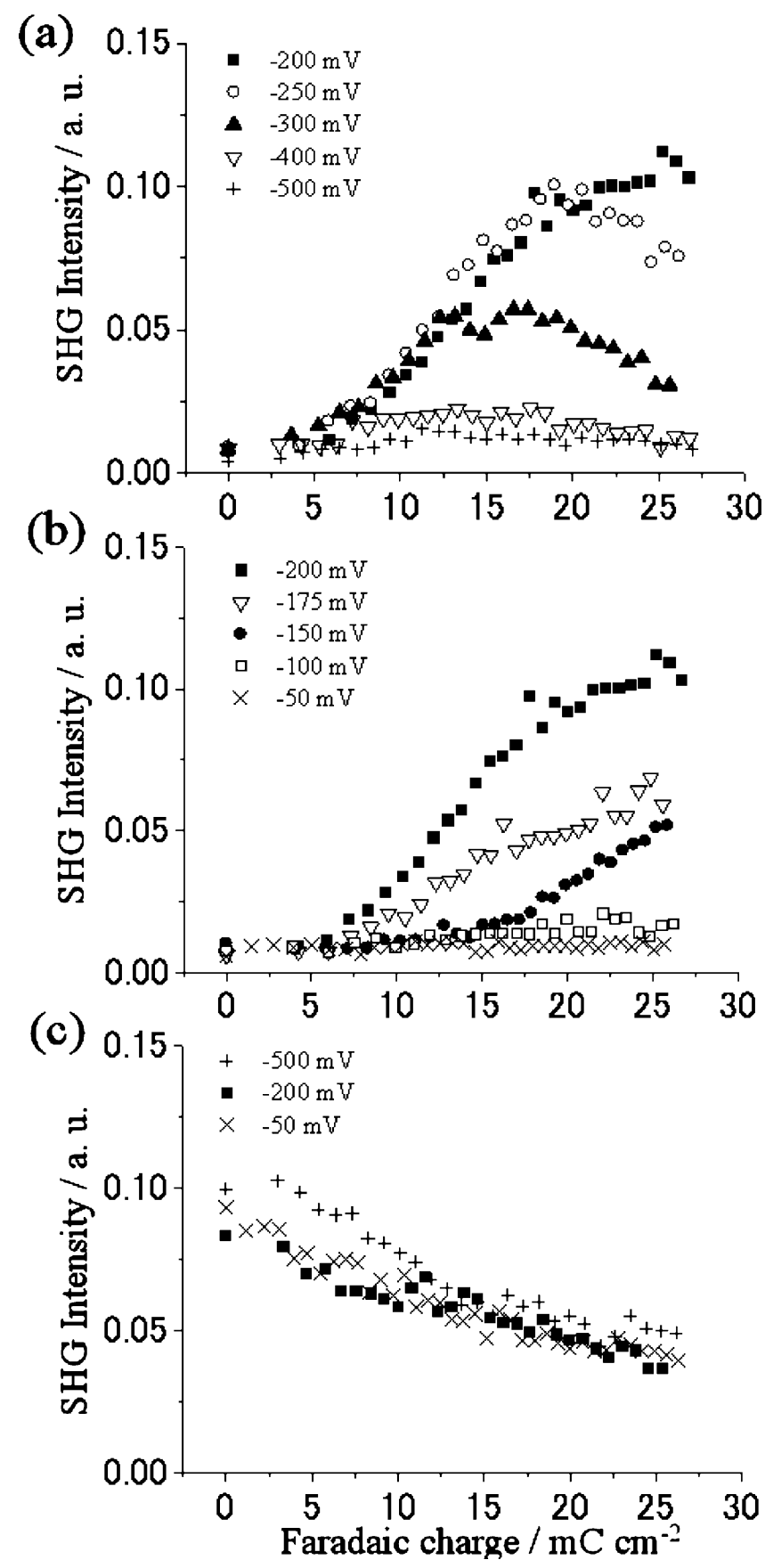

Figure 9. Faradaic charge dependences of p-in/p-out (a, b) and (c) $\mathrm{p}$-in/s-out SHG intensity at p-GaAs(001) electrode in $0.1 \mathrm{M} \mathrm{H}_{2} \mathrm{SO}_{4}$ solution containing $1 \mathrm{mM}$ of $\mathrm{CuSO}_{4}$ obtained with the potential step deposition. The deposition potentials are shown in the figure.

Ex situ AFM images of p-GaAs(001) surfaces after $\mathrm{Cu}$ has been deposited for $10 \mathrm{mC} \mathrm{cm} \mathrm{cm}^{-2}$ at (a) -200 and (b) $-500 \mathrm{mV}$ are shown in Figure 10. Relatively large $\mathrm{Cu}$ clusters with diameters of about $30-60 \mathrm{~nm}$ were studded at the deposition potential of $-200 \mathrm{mV}$. At the deposition potential of $-500 \mathrm{mV}$, a large number of smaller $\mathrm{Cu}$ clusters covered the entire GaAs surface, resulting in a relatively smooth surface. Faradaic chargedependent AFM images of p-GaAs(001) surfaces on which $\mathrm{Cu}$ deposition was carried out at the deposition potential of -200 $\mathrm{mV}$ are shown in Figure 11. The sizes of $\mathrm{Cu}$ clusters clearly increased with increase in deposition charge. On the other hand, the number of $\mathrm{Cu}$ deposits decreased with the progress of deposition because of the formation of larger clusters as a result of combination between the adjacent clusters. Compared with 

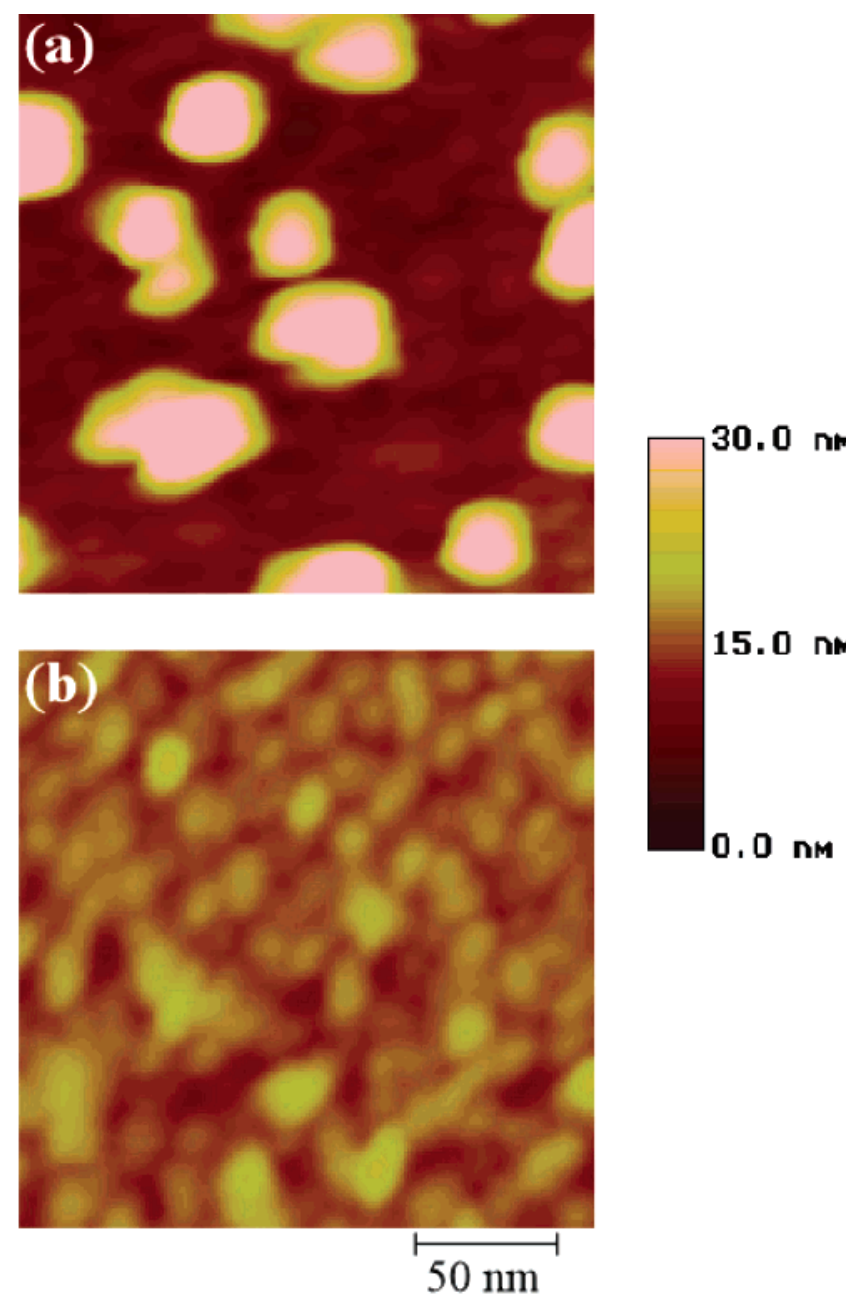

$15.0 \mathrm{nM}$

$0.0 \mathrm{~nm}$

Figure 10. AFM images of $\mathrm{Cu}$ nanoclusters deposited on p-GaAs(001) electrode at (a) -200 and (b) $-500 \mathrm{mV}$ after the deposition of $10 \mathrm{mC} \mathrm{cm}^{-2}$. Area: $200 \times 200 \mathrm{~nm}^{2}$.

the Faradaic charge dependence of surface SH intensity at the deposition potential of $-200 \mathrm{mV}$ (shown in Figure 9a or b as open squares), the surface $\mathrm{SH}$ intensity reached a maximum at the p-GaAs surface, which was dominantly covered by $\mathrm{Cu}$ clusters with diameters of about 40-50 nm.

Ex situ AFM measurements were also carried out at GaAs(001) surfaces on which $\mathrm{Cu}$ deposition was carried out at several deposition potentials, and the charge-dependent change in the cluster size distribution observed in $500 \times 500 \mathrm{~nm}^{2}$ AFM images was examined in detail for several deposition potentials. The charge-dependent cluster diameter distributions for the deposition potentials of (a) -100 , (b) -200 , (c) -300 , and (d) -500 $\mathrm{mV}$ are summarized in Figure 12. Typically, the lateral size distribution of $\mathrm{Cu}$ clusters deposited at larger overpotential $(-500 \mathrm{mV}$, Figure 12d) was not affected by increase in the $\mathrm{Cu}$ coverage since a large number of nucleation sites resulted in limitation for cluster growth in the lateral direction and onedirectional growth in the surface normal direction resulted in conservation of the lateral size distribution. This is consistent with results in the previous studies by optical reflectance/ scattering and AFM measurements. ${ }^{7}$ On the other hand, the lateral size distribution of $\mathrm{Cu}$ clusters deposited at a smaller overpotential ( $-100 \mathrm{mV}$, Figure 12a) strongly depended on the charge used for $\mathrm{Cu}$ deposition, and a broader distribution was observed on the surface with larger $\mathrm{Cu}$ coverage. Also, the number of $\mathrm{Cu}$ nanoclusters seems to be much smaller than that at more negative potentials. At $-300 \mathrm{mV}$ (Figure 12c), the
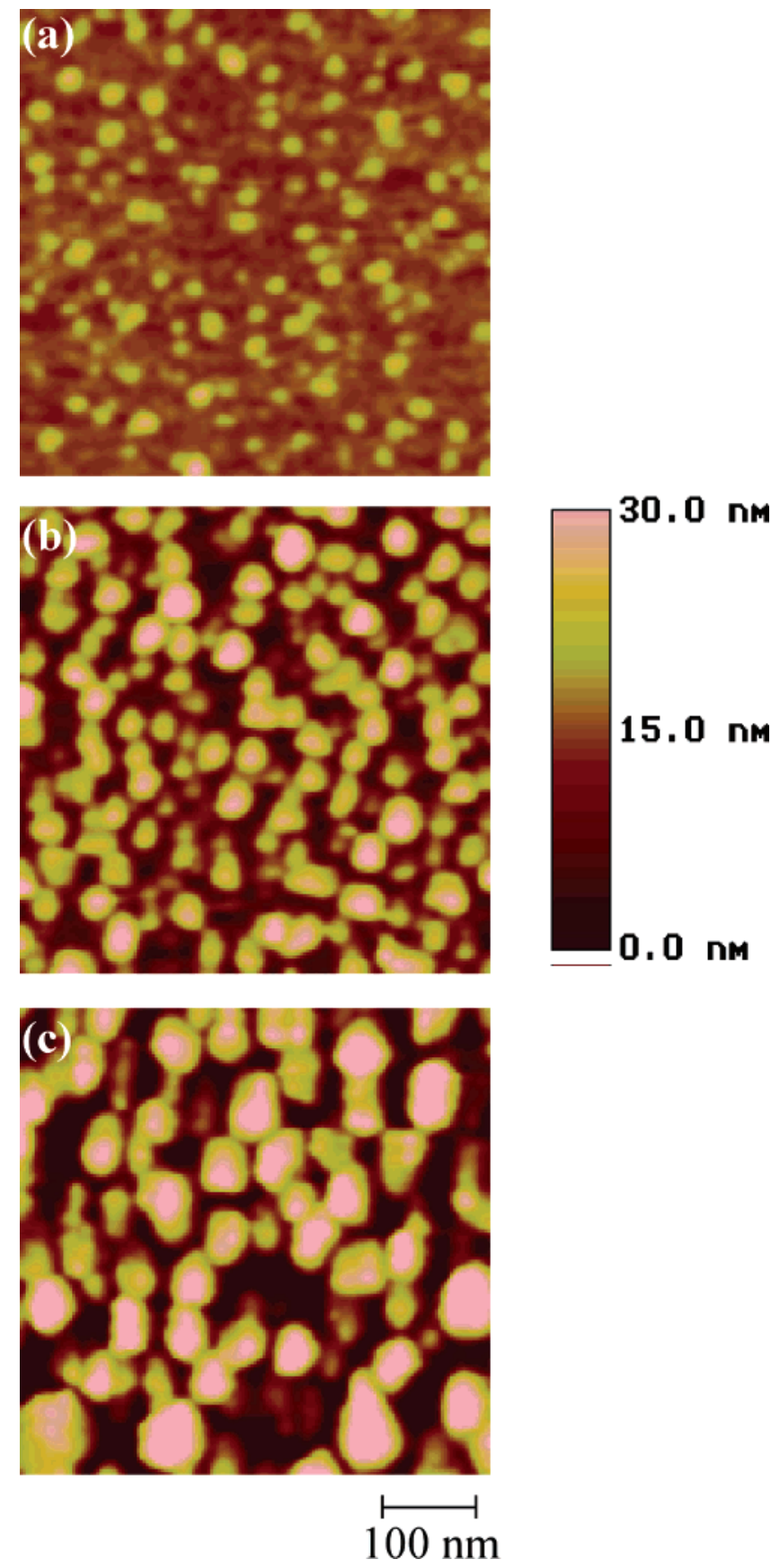

Figure 11. AFM images of $\mathrm{Cu}$ nanoclusters deposited on $\mathrm{p}-\mathrm{GaAs}$ (001) electrode at $-200 \mathrm{mV}$ after the deposition of (a) 5 , (b) 10 , and (c) $20 \mathrm{mC} \mathrm{cm}{ }^{-2}$. Area: $500 \times 500 \mathrm{~nm}^{2}$.

cluster size distribution obviously changed in the initial stage, but the change seems to be smaller for a larger amount of $\mathrm{Cu}$ deposition and becomes almost constant after deposition at a charge larger than $-15 \mathrm{mC} \mathrm{cm}^{-2}$. This may be due to change in the growth mode from three-dimensional to one-dimensional ${ }^{7}$ and will be discussed later. At $-200 \mathrm{mV}$ (Figure 12b), the peak in the cluster distribution showed a gradual change to a larger size direction. For this deposition potential, both an increase in cluster size distribution and a shift of peak position in the size distribution were observed. The shift of the peak position in the cluster size distribution may be dependent on the rate of $\mathrm{Cu}$ cluster growth. On the other hand, the cluster size distribution may be dependent on the number of nucleation sites. Larger nucleation sites at higher overpotentials cause limitation of the lateral area for $\mathrm{Cu}$ cluster growth and result in smaller size 
(a)

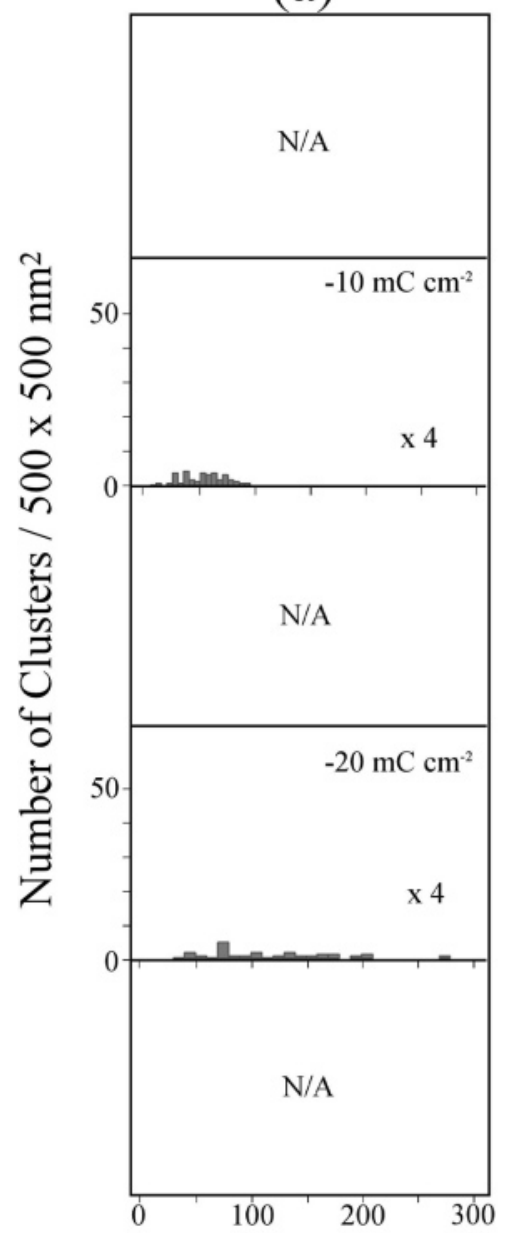

(b)

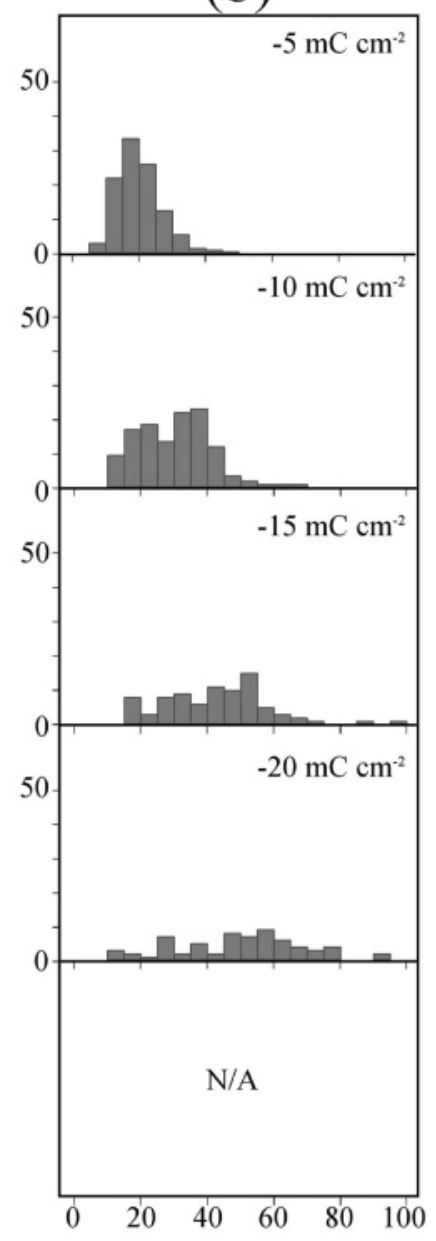

(c)

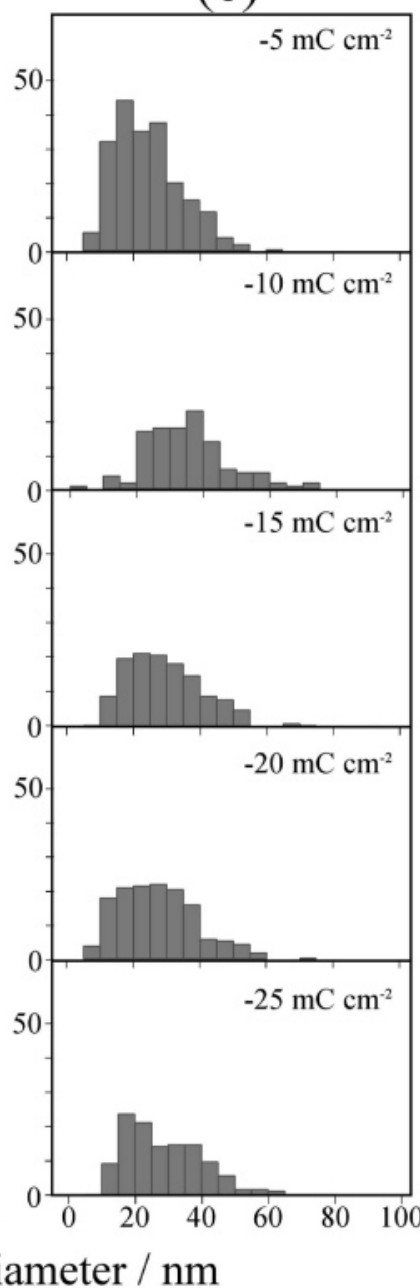

(d)

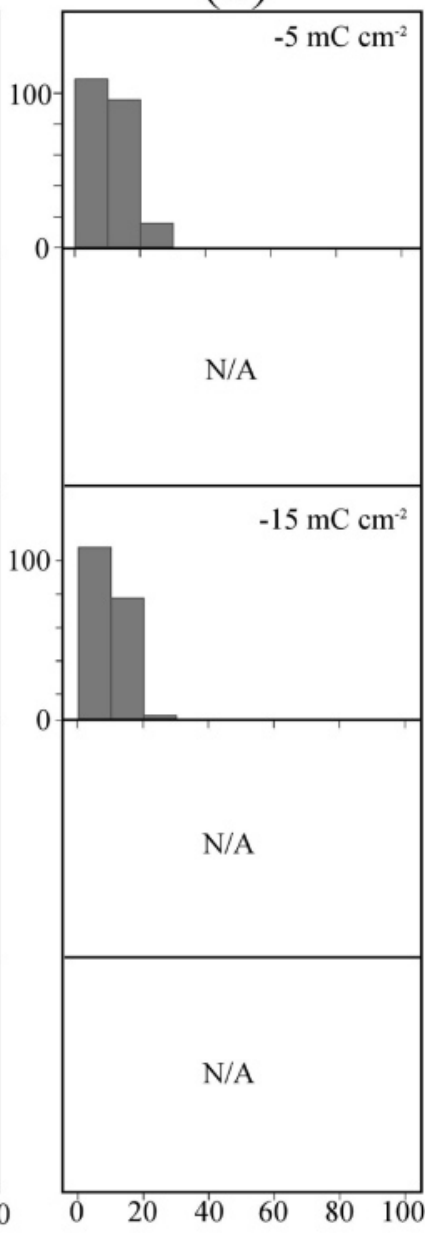

\section{Lateral Diameter / nm}

Figure 12. Lateral diameter distributions of $\mathrm{Cu}$ nanoclusters deposited on $\mathrm{p}-\mathrm{GaAs}(001)$ electrode at various deposition potentials; (a) -100, (b) -200 , (c) -300 , and (d) $-500 \mathrm{mV}$. Faradaic charge densities are shown as text in the figure.

distributions of $\mathrm{Cu}$ clusters. It was consequent speculations that a larger shift of the peak position and larger size distribution could be observed for positive deposition potentials of more than $-200 \mathrm{mV}$ and that a smaller shift of the peak position and smaller size distribution could be observed for negative deposition potentials of more than $-200 \mathrm{mV}$.

\section{Discussion}

\subsection{Modification of the SH-RA Pattern by $\mathrm{Cu}$ Deposition.} As mentioned in section 3.2, the p-in/p-out SH-RA pattern changed both in shape and amplitude during the $\mathrm{Cu}$ deposition with potential sweep, although the p-in/s-out SH-RA pattern changed only in amplitude. The change in the p-in/p-out SHRA pattern was attributed to both attenuation of bulk SHG and enhancement of surface SHG during $\mathrm{Cu}$ deposition. During $\mathrm{Cu}$ deposition, the decrease in the bulk SHG signal from p-GaAs(001) clearly depended on the coverage of $\mathrm{Cu}$ deposit as shown in Figure 6. On the other hand, the increase in the calculated surface SHG signal (Figure 6e) seemed to be independent of the surface coverage of $\mathrm{Cu}$ since the signal was still almost zero at the potential of $-300 \mathrm{mV}$ in the negative potential scan, where $-6 \mathrm{mC} \mathrm{cm}^{-2}$ of Faradaic charge was already consumed for the $\mathrm{Cu}$ deposition. Surface SHG showed an increase from the potential of $-300 \mathrm{mV}$, and continuous increase was observed up to the flow of the dissolution current of $\mathrm{Cu}$ deposit. Such a potential-dependent behavior of the optical signal was not observed at the same potential sweep rate in our previous work ${ }^{7}$ in which the reflectance and scattering signals of visible light during $\mathrm{Cu}$ deposition were utilized as optical probes. Since the reflectance is proportional to the thickness of the thin films and the scattering light intensity is proportional to both the square of the volume and the number of clusters, these are expected to have linear relations against the Faradaic charge, and it was confirmed experimentally in our previous work. ${ }^{7}$ However, the increase in surface SHG could not be correlated directly to film thickness of $\mathrm{Cu}$ or volume or number of $\mathrm{Cu}$ clusters. The mechanism of enhancement of the SHG signal should be clarified to apply SHG measurement as a tool for monitoring a surface event. The increase in surface SHG after the deposition of a certain amount of $\mathrm{Cu}$ can be attributed to the sensitivity of this signal to a more specific size or structure of the $\mathrm{Cu}$ deposit. A likely reason for the SHG enhancement is coupling with the surface plasmon induced in the $\mathrm{Cu}$ clusters.

4.2. Surface SHG Enhancement Induced by Specific Surface Morphology. Surface plasmon-assisted SHG enhancement from metal clusters has been reported for several systems, ${ }^{32-34}$ including Au nanocluster-deposited Si(111) surfaces. Suni and co-workers ${ }^{35-38}$ studied deposition kinetics of $\mathrm{Au}$ nanoclusters grown on $\mathrm{p}-\mathrm{Si}(111)$ by means of surface plasmon-enhanced SHG and noncontact AFM. They observed maxima in the time-dependent SHG profiles excited by light fields in a lateral direction during $\mathrm{Au}$ cluster formation, and 
the positions of the maxima depended on both the concentrations of gold-cyanide complex and the concentrations of HF in deposition solutions. From ex situ AFM images obtained at the sample surfaces emerged from deposition solutions, the maxima in the SHG profiles were attributed to gold clusters with lateral diameters of approximately $100 \mathrm{~nm} .{ }^{38}$ In their study, since the wavelength of SH light was also $532 \mathrm{~nm}$ and surface plasmon resonance occurs in the range of 510-540 nm for Au colloids of $2.5-10 \mathrm{~nm}$ in diameter, the SHG enhancement was directly associated with the electric field inside Au nanoclusters, which is enhanced by the surface plasmon resonance. In the present study, we should examine heights and shapes of $\mathrm{Cu}$ deposits in addition to the lateral sizes of $\mathrm{Cu}$ deposits since the $\mathrm{p}$-in/p-out polarization combination, which contained electric fields perpendicular to the surface, was used for the surface SHG measurements.

If we just compare the SHG intensity profile in Figure 9a and the charge-dependent AFM images at the deposition potential of $-200 \mathrm{mV}$ in Figure 11, SHG enhancement depending on the lateral size of $\mathrm{Cu}$ clusters seems to be reasonable, and we can simply speculate that the SHG signal is dependent on the number of $\mathrm{Cu}$ clusters with diameters of about $50 \mathrm{~nm}$. However, the results shown in Figures 9a and 12c cannot be explained by this scenario. For example, the formation of $\mathrm{Cu}$ clusters with diameters larger than $50 \mathrm{~nm}$ was not observed at the deposition potential of $-300 \mathrm{mV}$, where the maximum in the SHG profile was clearly observed in Figure 9a. Although the number of $\mathrm{Cu}$ clusters was much less, $\mathrm{Cu}$ clusters with diameters larger than $50 \mathrm{~nm}$ were observed at the deposition potential of $-100 \mathrm{mV}$, where no SHG change was observed. Thus, SHG enhancement cannot be explained only by the lateral diameter of $\mathrm{Cu}$ nanoclusters, and a different mechanism to explain the SHG enhancement should be required.

The correlation between the height of $\mathrm{Cu}$ clusters on GaAs and the $\mathrm{SH}$ intensity was also examined. In Figure 13, Faradaic charge dependences of the apparent height distribution of $\mathrm{Cu}$ deposits at the deposition potentials of (a) -200 and (b) -300 $\mathrm{mV}$ obtained from ex situ AFM images are shown. At -200 $\mathrm{mV}$, the height of $\mathrm{Cu}$ deposits became larger with increase in the deposition charge, and the height distribution gradually became broader, corresponding to the charge-dependent SHG profile (Figure 9a). On the other hand, at $-300 \mathrm{mV}$, the height of deposits seemed to depend on the deposition charge in the initial stage, but both the apparent heights and their distribution no longer changed for deposition charges of more than -15 $\mathrm{mC} \mathrm{cm}{ }^{-2}$. Although the Faradaic charge-dependent SHG profile (Figure 9a) at this deposition potential showed attenuation from this deposition charge, a direct correlation cannot be found.

A remaining factor concerning the surface plasmon-assisted SHG enhancement is the shape of the $\mathrm{Cu}$ nanoclusters. As shown in the papers dealing with the absorption spectrum of cylindrical particles, the shape of the noble metal particles strongly affected the absorption spectrum. ${ }^{39,40}$ However, there is no direct relationship between the aspect ratio of $\mathrm{Cu}$ nanoclusters and the SHG enhancement (see Supporting Information), although the shape of the $\mathrm{Cu}$ nanoclusters always seems to be flattened or oblate on the samples where the SHG enhancement was observed.

As mentioned above, none of the parameters (lateral diameter, height, and aspect ratio) of $\mathrm{Cu}$ nanoclusters can be directly correlated with the Faradaic charge-dependent SHG profiles. However, it should be noted that all of the parameters of $\mathrm{Cu}$ nanoclusters at the deposition potential of $-300 \mathrm{mV}$ changed their behavior at around $-15 \mathrm{mC} \mathrm{cm}^{-2}$, where the enhanced
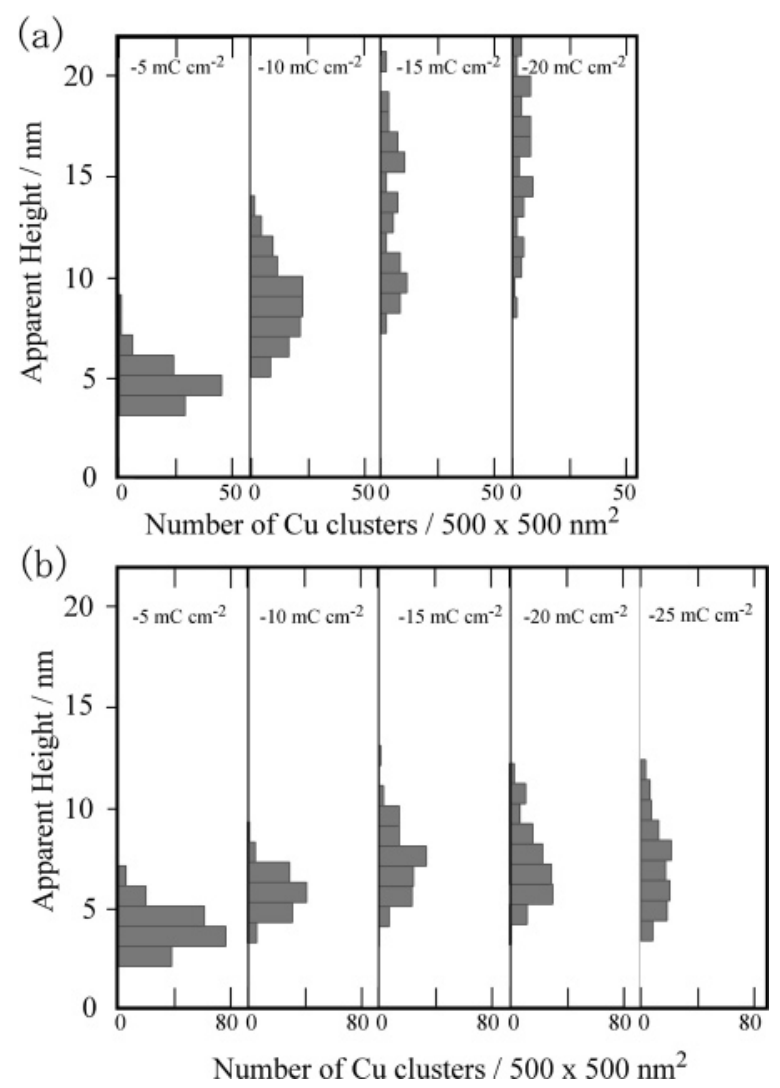

Figure 13. Faradaic charge dependences of height distributions of $\mathrm{Cu}$ nanoclusters deposited on $\mathrm{p}-\mathrm{GaAs}(001)$ electrode at the deposition potentials of (a) -200 and (b) $-300 \mathrm{mV}$.

SH intensity started to decrease. All of the distributions of lateral diameter, height, and aspect ratio were almost the same at the surfaces with deposition charge from -15 to $-25 \mathrm{mC} \mathrm{cm}^{-2}$, as shown in Figures 12 and 13, although the amount of $\mathrm{Cu}$ deposit increased. This fact indicates that change in growth mode from three-dimensional to one-dimensional growth in the surface normal direction occurred at a deposition charge between -10 and $-15 \mathrm{mC} \mathrm{cm}{ }^{-2}$. In other words, all the surface of GaAs was covered with $\mathrm{Cu}$ deposits at that deposition charge, and the surface morphology was kept during further deposition. Thus, at the deposition potential of $-300 \mathrm{mV}$, the number of isolated $\mathrm{Cu}$ nanoclusters started to decrease with $\mathrm{Cu}$ film formation from the deposition charge of $-15 \mathrm{mC} \mathrm{cm}^{-2}$, and then the decline of surface plasmon-enhanced $\mathrm{SH}$ intensity started. This speculation is supported by the fact that the enhancement of SH intensity was not observed at the deposition potential of $-500 \mathrm{mV}$, where a continuous $\mathrm{Cu}$ film was formed from the initial stage. On the other hand, at the deposition potential of $-200 \mathrm{mV}$, distribution changes in height and lateral diameter showed that isolated $\mathrm{Cu}$ nanoclusters remained and the three-dimensional growth of $\mathrm{Cu}$ deposits continued even at the deposition charge of $-20 \mathrm{mC} \mathrm{cm}{ }^{-2}$. Of course, at the deposition potential of $-100 \mathrm{mV}$, the isolated $\mathrm{Cu}$ nanoclusters were formed, but the number of large $\mathrm{Cu}$ nanoclusters was smaller, as shown in Figure 12a, and then the contribution to the SHG signal was negligible. From the above discussion, we assumed that $\mathrm{SH}$ enhancement could be observed at GaAs surfaces with a number of isolated, relatively large $\mathrm{Cu}$ nanoclusters with flattened shapes.

4.3. Surface Plasmon-Induced Enhancement of Flattened Cu Clusters. Optical plasmon resonance in noble metal nanoparticles was first theoretically described by Mie by solving Maxwell's equations for a radiation field interacting with a 
spherical metal particle under appropriate boundary conditions. ${ }^{39}$ The surface plasmon resonance in the absorption spectrum was simulated by using Mie's theory in the wavelength range from 550 to $600 \mathrm{~nm}$ for $\mathrm{Cu}$ particles with sizes from 1 to $20 \mathrm{~nm}$, and a plasmon absorption peak at $558 \mathrm{~nm}$ appears progressively upon increasing the size of $\mathrm{Cu}$ particles. ${ }^{41,42}$ This plasmon resonant wavelength is far from the present fundamental wavelength (1064 nm) and slightly longer than the SH wavelength (532 $\mathrm{nm})$. Thus, resonant enhancement cannot occur in such measurement conditions, although the surface plasmon resonance depends on the shape, size, and density of clusters and on the dielectric function of the surrounding medium.

In the present study, as was clearly observed by AFM images, flattened or oblate $\mathrm{Cu}$ ellipsoids were formed. For prolate and oblate spheroidal particles, Gans extended Mie's theory. Here, the visible absorption spectra of oblate or flattened $\mathrm{Cu}$ ellipsoids with different aspect ratios, $R=r / l$, where $r$ and $l$ are the lateral diameter and the apparent height of a $\mathrm{Cu}$ nanocluster, respectively, are simulated by Gans' theory. The extinction coefficient $\gamma$ of small randomly oriented particles is ${ }^{39}$

$$
\gamma=N V \frac{2 \pi \epsilon_{\alpha}^{3 / 2}}{3 \lambda} \sum_{j} \frac{\left(\frac{1}{P_{\mathrm{j}}^{2}}\right) \epsilon_{2}}{\left(\epsilon_{1}+\frac{1-P_{\mathrm{j}}}{P_{\mathrm{j}}} \epsilon_{\alpha}\right)^{2}+\epsilon_{2}^{2}}
$$

where $N$ is the number of particles per unit volume, $V$ is the volume of each particle, $\epsilon_{\alpha}$ is the dielectric constant of the surrounding medium, $\lambda$ is the wavelength of the interacting light, $\epsilon_{1}$ and $\epsilon_{2}$ are the real and imaginary parts of the material dielectric function, respectively, and $P_{\mathrm{j}}$ is the depolarization factor corresponding to each of the axes, A, B, and C, of the particle. For oblate or flattened ellipsoids $(\mathrm{A}=\mathrm{C}=r>\mathrm{B}=$ 1), this factor has been defined as

$$
P_{\mathrm{B}}=\frac{1}{e^{2}}\left[1-\left(\frac{1-e^{2}}{e^{2}}\right)^{1 / 2} \sin ^{-1} e\right]
$$

and

$$
P_{\mathrm{A}}=P_{\mathrm{C}}=\frac{1-P_{\mathrm{B}}}{2}
$$

where

$$
e=\left(\frac{A^{2}-B^{2}}{A^{2}}\right)^{1 / 2}
$$

If the ellipsoids are oriented in a parallel manner, the Gans equations are applicable without any correction. ${ }^{39}$ With the known values for the complex dielectric constant of copper, ${ }^{43}$ eq 2 is plotted in Figure 14a and b for flattened $\mathrm{Cu}$ nanoparticles with different aspect ratios and median dielectric constant fixed to a value of 2.0. It can be seen that increase in the aspect ratio causes only a red-shift in the frequency of the plasmon resonance peak. The mean aspect ratios obtained for $\mathrm{Cu}$ nanoclusters deposited at -200 or $-300 \mathrm{mV}$ are around $3-10$, and these values can only result in a plasmon resonant peak in the wavelength region from 600 to $900 \mathrm{~nm}$. Also, no blue-shift of the plasmon peak was observed for $\mathrm{Cu}$ nanoclusters. Thus, the plasmon-enhanced SHG caused by resonance between the plasmon in flattened $\mathrm{Cu}$ ellipsoids and the $\mathrm{SH}$ photon of 532 nm cannot occur, and our first assumption failed. However, the
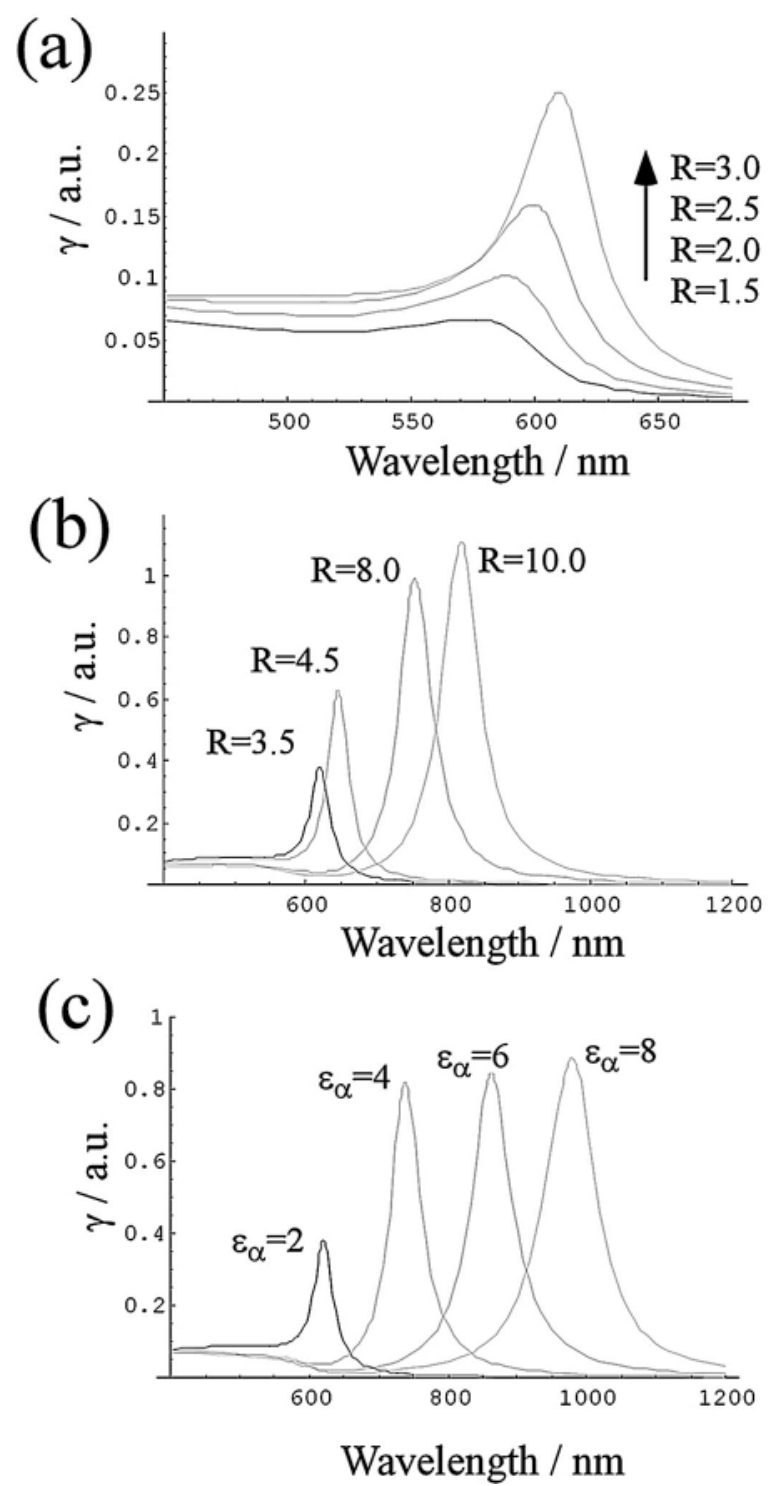

Figure 14. Results of simulation for extinction coefficient, $\gamma$, of $\mathrm{Cu}$ nanoclusters. $(\mathrm{a}, \mathrm{b})$ : Aspect ratio dependences for $\mathrm{Cu}$ nanoclusters with aspect ratio, $R$, from 1.5 to 10.0. $\epsilon_{\alpha}=2.0$. (c) Surrounding dielectric constant dependence for $\mathrm{Cu}$ nanoclusters with aspect ratio of $R=3.5$. $\epsilon_{\alpha}=2.0-8.0$.

possibility that the position of the plasmon resonant peak strongly depends on the surrounding dielectric constant, $\epsilon_{\alpha}$, arises. In Figure 14c, the spectra of $\mathrm{Cu}$-flattened ellipsoids with an aspect ratio of 3.5 , which is a typical value for $\mathrm{Cu}$ deposits at the deposition potential of $-200 \mathrm{mV}$, are plotted for several values of $\epsilon_{\alpha}$. As shown in this figure, the plasmon resonant peak of $\mathrm{Cu}$ ellipsoids shifts in a longer wavelength with increase in $\epsilon_{\alpha}$ and finally reaches more than $1 \mu \mathrm{m}$. In the present system, the value of $\epsilon_{\alpha}$ cannot be easily estimated since the $\mathrm{Cu}$ ellipsoids are located at the interface between the aqueous solution $\left(\epsilon_{\alpha} \approx\right.$ 1.79 ) and GaAs substrate. The dielectric function of GaAs is a complex number depending on the frequency and differs greatly for $\lambda=532 \mathrm{~nm}(16.65+\mathrm{i} 2.35)$ and $\lambda=1064 \mathrm{~nm}(11.94)^{44}$ and obviously much larger than that of the aqueous solution. Although estimation of the dielectric function for surroundings of $\mathrm{Cu}$ nanoclusters seems impossible, a red-shift of the plasmon resonant peak for $\mathrm{Cu}$ ellipsoids deposited on dielectric material is expected to be possible. Therefore, resonant enhancement of SHG may be possible by coupling between a plasmon wave 
induced in the flattened $\mathrm{Cu}$ ellipsoids on the GaAs substrate and the fundamental light of $1.064 \mu \mathrm{m}$.

The SHG behavior at the deposition potential of $-300 \mathrm{mV}$ in Figure 9a indicated the impact of change in the dielectric function of surroundings, $\epsilon_{\alpha}$, on the SHG resonance. As mentioned above, for this deposition potential, the monotonically increasing SHG intensity started to decline after the deposition charge of $-15 \mathrm{mC} \mathrm{cm}^{-2}$, at which all of the surface of GaAs seemed to be covered by $\mathrm{Cu}$. This means that $\mathrm{Cu}$ flattened clusters are no longer located on the GaAs but are located on the continuous $\mathrm{Cu}$ thin film, and then the resonant frequencies of surface plasmon in $\mathrm{Cu}$ nanoclusters should, therefore, change. At the deposition potential of $-250 \mathrm{mV}$, it is speculated that the formation of a continuous $\mathrm{Cu}$ film caused the SHG decline beyond the deposition charge of $-20 \mathrm{mC} \mathrm{cm}^{-2}$.

An additional factor causing the red-shift of the plasmon resonant peak of $\mathrm{Cu}$ nanoclusters can be considered. According to Maxwell-Garnett theory, if the volume of an active medium (e.g., metal) in the sample is large compared to the volume of surrounding medium, the plasmon peak shows a red-shift. ${ }^{39}$ Such a plasmon frequency shift was shown by simulation of the absorption spectra of $\mathrm{Ag}-\mathrm{SiO}_{2}$ films. ${ }^{40}$ Huang et al. experimentally found that the dispersibility of gold nanoparticles in various solvents caused modification in the shape of visible absorption spectra. ${ }^{45}$ In their study, more dense Au nanoparticles resulted in broadening and red-shifting in the plasmon absorption peak. In the present case, the dispersibility of $\mathrm{Cu}$ nanocluster became smaller when the $\mathrm{Cu}$ clusters became larger in the lateral direction at smaller overpotentials, and this may also play an important role in the resonant enhancement of SHG signals from $\mathrm{Cu}$ nanoculsters.

From the above discussion, it can be concluded that SHG enhancement should occur at a GaAs surface with isolated flattened $\mathrm{Cu}$ nanoclusters with relatively large volume because of resonant coupling between the surface plasmon and fundamental 1064-nm light. However, both the difficulties in estimating both the actual value of $\epsilon_{\alpha}$ and the dispersibility of $\mathrm{Cu}$ nanoclusters at the surface prevent us from expecting the most SHG active size, shape, and aspect ratio of the $\mathrm{Cu}$ nanoclusters.

4.4. Attenuation of Bulk SHG by $\mathrm{Cu}$ Deposition. As shown in Figure 9c, the p-in/s-out SHG signal from bulk GaAs decreased with the progress of $\mathrm{Cu}$ deposition. Although the surface morphology of the $\mathrm{Cu}$ deposit depended on the deposition potential as shown in the AFM images in Figure 10, attenuation of the SHG signal from bulk GaAs seemed to depend only on the amount of $\mathrm{Cu}$ deposits at each deposition potential. This seems strange, because even the linear optical signals were strongly affected by surface morphology in our previous study. ${ }^{7}$ However, the nature of the nonlinear optical effect should be considered, to understand the present results. SH light from GaAs bulk is the result of radiation by the second-order nonlinear dipole induced in the bulk of GaAs and is dependent on the power of the incident electric field. Dispersed large $\mathrm{Cu}$ nanoclusters, which are formed at more positive potentials such as $-100 \mathrm{mV}$, cause optical scattering of both incident fundamental photons and generated $\mathrm{SH}$ photons. On the other hand, close-packed small $\mathrm{Cu}$ nanoclusters, which are formed at more negative potentials such as $-500 \mathrm{mV}$, behave as a $\mathrm{Cu}$ thin film and strongly reflect the fundamental light and absorb $\mathrm{SH}$ photons. Both the scattering and reflection of a fundamental beam caused weakening of the electric field reaching in bulk GaAs. Also, both the scattering and absorption of SH photons coming from bulk GaAs caused weakening of the total SHG signal. In our previous paper, ${ }^{7}$ the tradeoff correlation between the scattering and the reflectance signals in the same system was shown. Therefore, the coincidence in the Faradaic chargedependent decrease of SHG signal from GaAs bulk at any deposition potential may be considered to be a result of masking both the fundamental and $\mathrm{SH}$ lights by $\mathrm{Cu}$ deposits, although the morphology of the $\mathrm{Cu}$ deposit affected the way in which the light wave was masked.

\section{Conclusion}

It is demonstrated that the SHG method can be used to monitor surface phenomena even when the bulk SHG signal is significant. While bulk SHG signals from p-GaAs(001) monotonically decreased with the amount of electrodeposited $\mathrm{Cu}$ on the surface, increase in surface SHG was observed only when discontinuous flattened $\mathrm{Cu}$ nanoclusters with relatively large volume (diameter $>30 \mathrm{~nm}$, height $>5 \mathrm{~nm}$ ) were formed on the $\mathrm{GaAs}(001)$ surface. Both the coverage-dependent decrease in bulk SHG and the morphology-dependent increase in surface SHG caused modification of the $\mathrm{p}$-in/p-out SH-RA pattern during $\mathrm{Cu}$ electrodeposition under potential cycling. In the case of potentiostatic deposition, the morphology of $\mathrm{Cu}$ seemed to be well controlled as a function of deposition potential, and factors involved in the enhancement of surface SHG by resonant coupling of the surface plasmon with near-infrared fundamental light were estimated. Because of the difficulties in controlling the size distribution, the dispersibility, and the shapes of $\mathrm{Cu}$ nanoclusters uniformly, it was not possible to determine the detailed conditions concerning surface plasmon-induced SHG resonant enhancement. To determine rigid resonant conditions, the present SHG measurement system is now upgraded to an in situ SHG spectroscopic measurement system in a broad frequency region and will be a simple and powerful tool to characterize surface nanostructures.

Acknowledgment. We are grateful to Mr. H. Fujisawa (Mitsubishi Chemical Co.) for providing us with the GaAs wafers. This work was partially supported by a Grant-in-Aid for Scientific Research on Priority Areas "Molecular Nano Dynamics" from the Ministry of Education, Culture, Sports, Science, and Technology (MEXT), Japan. This work was also supported by CREST of JST (Japan Science and Technology Agency).

Supporting Information Available: Calibration methods for AFM measurements and a figure showing charge-dependent change in the aspect ratio distribution of $\mathrm{Cu}$ nanoclusters (Figure $1 \mathrm{~S})$. This material is available free of charge via the Internet at http://pubs.acs.org.

\section{References and Notes}

(1) Koinuma, M.; Uosaki, K. Electrochim. Acta 1995, 40, 1345.

(2) Koinuma, M.; Uosaki, K. J. Electroanal. Chem. 1996, 409, 45.

(3) Koinuma, M.; Uosaki, K. Surf. Sci. 1996, 358, 565.

(4) Koinuma, M.; Hirae, T.; Matsumoto, Y. J. Mater. Res. 1998, 13, 837.

(5) Tamura, K.; Oyanagi, H.; Kondo, T.; Koinuma, M.; Uosaki, K. J. Phys. Chem. B 2000, 104, 9017.

(6) Kondo, T.; Tamura, K.; Koinuma, M.; Oyanagi, H.; Uosaki, K. Chem. Lett. 1997, 761.

(7) Tamura, K.; Kondo, T.; Uosaki, K. J. Electrochem. Soc. 2000, 147, 3356.

(8) Scherb, G.; Kolb, D. M. J. Electroanal. Chem. 1995, 396, 151.

(9) Eisenthal, K. B. Annu. Rev. Phys. Chem. 1992, 43, 627.

(10) Heinz, T. F.; Reider, G. A. TrAC, Trends Anal. Chem. 1989, 8, 235.

(11) McGilp, J. F. J. Phys. D: Appl. Phys. 1996, 29, 1812.

(12) Richmond, G. L.; Robinson, J. M.; Shannon, V. L. Prog. Surf. Sci. 1988, 28,1 .

(13) Shen, Y. R. Nature 1989, 337, 519.

(14) Corn, R. M.; Higgins, D. A. Chem. Rev. 1994, 94, 107. 
(15) Yagi, I.; Nakabayashi, S.; Uosaki, K. J. Phys. Chem. B 1997, 101 7414.

(16) Yagi, I.; Nakabayashi, S.; Uosaki, K. Surf. Sci. 1998, 406, 1. 2677.

(18) Awatani, T.; Yagi, I.; Noguchi, H.; Uosaki, K. J. Electroanal. Chem. 2002, 524-525, 184.

(19) Germer, T. A.; Kolasinski, K. W.; Stephenson, J. C.; Richter, L. J. Phys. Rev. B: Solid State 1997, 55, 10694.

(20) Stehlin, H.; Feller, M.; Guyot-Sionnest, P.; Shen, Y. R. Opt. Lett. 1988, 13, 389 .

(21) Yamada, C.; Kimura, T. Jpn. J. Appl. Phys. 1995, 34, 1102.

(22) Yamada, C.; Kimura, T. Phys. Rev. Lett. 1993, 70, 2344.

(23) Kimura, T.; Yamada, C. Jpn. J. Appl. Phys. 1995, 34, 1498.

(24) Kimura, T.; Yamada, C. J. Cryst. Growth 1995, 150, 92.

(25) Yamada, C.; Kimura, T. Phys. Rev. B: Solid State 1994, 49, 14372.

(26) Tanaka, H.; Mizutani, G.; Ushioda, S. Surf. Sci. 1998, 404, 533.

(27) Hollering, R. W. J. Opt. Commun. 1992, 90, 142.

(28) Yamauchi, T.; Sonoda, Y.; Sakamoto, K.; Ushioda, S.; Sano, H.; Sakai, J.; Mizutani, G. Surf. Sci. 1996, 363, 385.

(29) Takebayashi, M.; Mizutani, G.; Ushioda, S. Opt. Commun. 1997, $133,116$.

(30) Sano, H.; Shimizu, T.; Mizutani, G.; Ushioda, S. J. Appl. Phys. 2000, 87, 1614 .
(31) Lazarescu, V.; Lazarescu, M. F.; Jones, H.; Schmickler, W. J. Electroanal. Chem. 2004, 567, 257.

(32) Shen, Y. Z.; Swiatkiewicz, J.; Winiarz, J.; Markowicz, P.; Prasad, P. N. Appl. Phys. Lett. 2000, 77, 2946.

(33) Antoine, R.; Pallarin, M.; Palpant, B.; Broyer, M.; Prevel, B.; Galletto, P.; Brevet, P. F.; Girault, H. H. J. Appl. Phys. 1998, 84, 4532.

(34) Krein-Wiele, J.-H.; Simon, P.; Rubahn, H.-G. Phys. Rev. Lett. 1998 80,45 .

(35) Srinivasan, R.; Suni, I. I. Surf. Sci. 1998, 408, L 698.

(36) Srinivasan, R.; Suni, I. I. J. Electrochem. Soc. 1999, 146, 570.

(37) Srinivasan, R.; Tian, Y.; Suni, I. I. Surf. Sci. 2001, 490, 308.

(38) Rossiter, C.; Suni, I. I. Surf. Sci. 1999, 430, L553.

(39) Papavassiliou, G. C. Prog. Solid State Chem. 1980, 12, 185

(40) Skillman, D. C.; Berry, C. J. Chem. Phys. 1968, 48, 3297.

(41) Kapoor, S.; Palit, D. K.; Mukherjee, T. Chem. Phys. Lett. 2002, $355,383$.

(42) Lisiecki, I.; Billoudet, F.; Pileni, M. P. J. Phys. Chem. 1996, 100, 4160 .

(43) Johnson, P. B.; Christy, R. W. Phys. Rev. B: Solid State 1972, 6, 4370 .

(44) Zollner, S. J. Appl. Phys. 2001, 90, 515

(45) Huang, S.; Minami, K.; Sakaue, H.; Shingubara, S.; Takahagi, T. J. Appl. Phys. 2002, 92, 7486. 